\title{
The continuum theory of shear localization in two-dimensional foam
}

\author{
Denis Weaire, Joseph D. Barry and Stefan Hutzler \\ School of Physics, Trinity College Dublin, Ireland \\ E-mail: stefan.hutzler@tcd.ie
}

\begin{abstract}
We review some recent advances in the rheology of two-dimensional liquid foams, which should have implications for three-dimensional foams, as well as other mechanical systems that have a yield stress. We focus primarily on shear localization under steady shear, an effect first highlighted in an experiment by Debrégeas et al. A continuum theory which incorporates wall drag has reproduced the effect. Its further refinements are successful in matching results of more extensive observations and make interesting predictions regarding experiments for low strain rates, and non-steady shear. Despite these successes, puzzles remain, particularly in relation to quasistatic simulations. The continuum model is semiempirical: the meaning of its parameters may be sought in comparison with more detailed simulations and other experiments. The question of the origin of the Herschel-Bulkley relation is particularly interesting.
\end{abstract}

PACS numbers: 47.57.Bc Foams and emulsions, 83.60.Fg Shear rate dependent viscosity, 83.10.Ff Continuum mechanics, 83.80.Iz, Emulsions and foams.

\section{Introduction}

Precisely how do foams flow? This question was posed in a review article by Kraynik at the outset of the modern development of the subject [1]. It is of direct practical relevance to industry, and a useful focus of attention in the study of basic rheology. A liquid foam can stand as a prototype for a wide class of substances - those that have a yield stress, behaving as solids under low stress and liquids under high stress. 


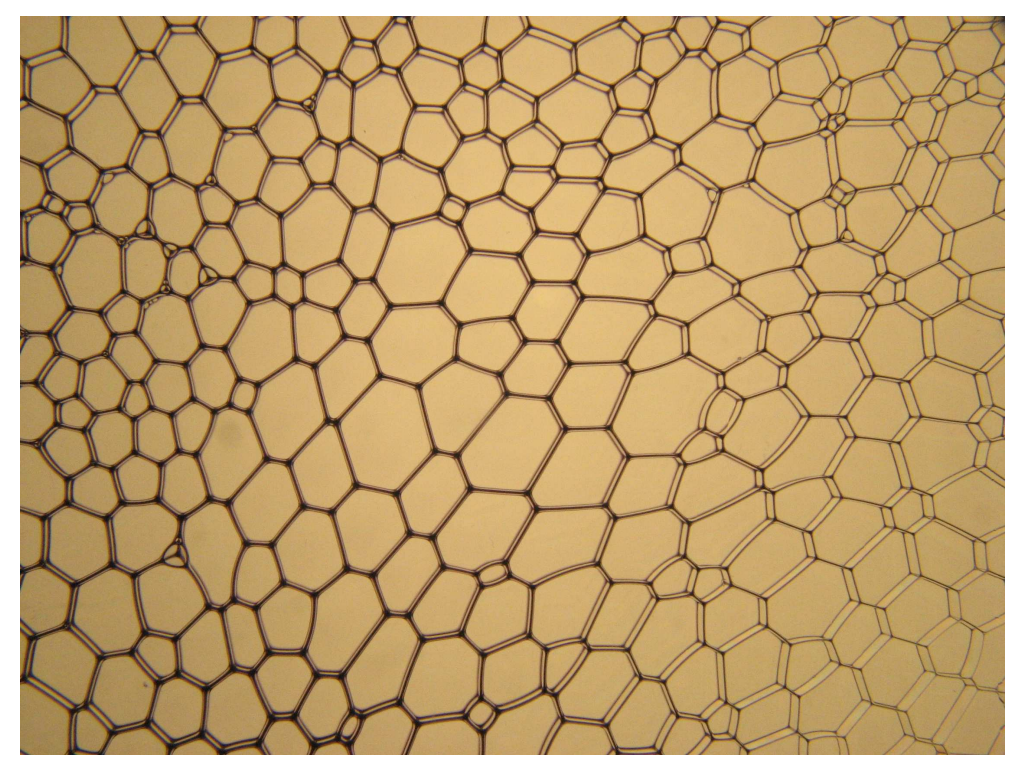

Figure 1. Recent progress in foam rheology was instigated by experiments with two-dimensional foams, obtained for example by squeezing foam between two glass plates, as in this photograph.

They include many pastes, powders, suspensions, gels and emulsions. These are generally disordered aggregates of individual entities (particles, droplets, bubbles). They may be called Bingham fluids, by reference to a particular (and rarely accurate) theoretical representation of their dual solid/liquid character.

The particular appeal of foam lies in its clearly defined and simple local structure, whose rearrangements under shear may be straightforwardly characterized. It may be realized, visualized and simulated with relative ease. All of this is especially true of a two-dimensional foam, a single layer of interacting bubbles, as shown in figure 1. Such a foam may be formed in various ways; it is sometimes important to distinguish between them. It may be prepared by trapping the bubbles between two plates, or by letting them float on liquid, with or without a confining plate on top. These alternatives are illustrated in figure 2.

The first of these options (Figure 2(c)) was popularized by Smith [2]. He was originally motivated by a study of grain growth in metals. The freely floating foam (Figure 2(a)) is Bragg's soap raft, which he introduced as a model for atomic arrangements in crystals [3]. The intermediate option (Figure 2(b)) was introduced 


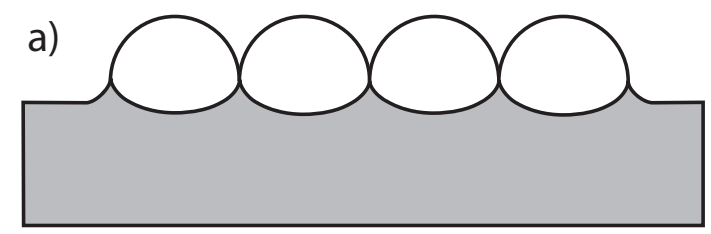

b)

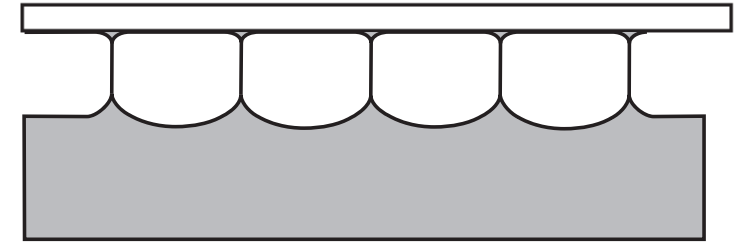

c)

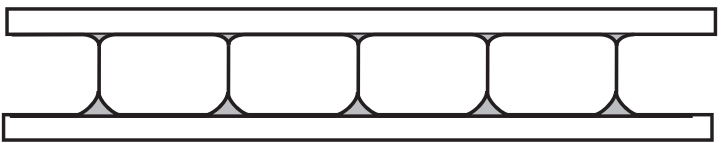

Figure 2. Three types of $2 \mathrm{D}$ foams: (a) monolayer of air bubbles sitting at an air/liquid interface (Bragg raft); (b) bubbles floating in liquid under a glass plate; (c) bubbles confined between two glass plates. There are large effects due to the drag associated with motion relative to solid boundaries in both (b) and (c).

by Earnshaw [4] and by Vaz and Fortes [5].

Of course, as Figure 2 makes plain, none of these foams is truly two-dimensional; nevertheless 2D models often suffice to describe their properties. Particularly when statics (or quasistatics) is all that is at stake, the differences between the three kinds of $2 \mathrm{D}$ foam sample may often be disregarded. However, that does not appear to be the case for 2D foam rheology, which is the topic of this review. It matters a great deal whether a confining plate is present as in Figures 2 (b) and (c).

At the end of a book that summarized what was known about basic foam properties up to the year 2000, Weaire and Hutzler emphasised that dynamic properties were very important yet hardly understood at all [6]. Since that time several groups have made important initial contributions to foam rheology. They include among others the groups associated with Durian [7, 8, 9], Cantat [10, 11, 12], Graner [13, 14, 15, 16, 17] and Vandewalle [18], who have provided a wealth of 
examples of 2D foam flow, and models that represent it. Models of bubble-bubble interactions with relevance also to $3 \mathrm{D}$ were developed in the group of Denkov $[19,20,21]$.

With benefit of hindsight, one may see that an attack on dynamical problems could have been pursued in 2D foams much earlier. It was inhibited by a vague sense that the $2 \mathrm{D}$ foam might not correspond well to the $3 \mathrm{D}$ foam whose analysis was the ultimate goal. This doubt is quite reasonable, as we shall see: nevertheless it is possible to learn a great deal from the $2 \mathrm{D}$ case, if it is properly understood.

In 2001 the subject was brought to a sharp focus in an experiment by Debrégeas et al. [22]. A monolayer of bubbles, confined between two plates (as in figure 2 (c)), was introduced in a 2D cylindrical Couette viscometer of an original design. The phenomenon highlighted by the experiment was shear localization, or shear banding. When the inner cylinder was rotated, the associated shearing motion of the bubbles was restricted to a narrow region close to it. With benefit of hindsight one may discern something similar in earlier work, but here it was clearly exposed and quantified, demanding explanation. The ensuing debate has drawn in several experimental and theoretical groups, and has reached such a stage that the present topical review is justified. That is to say, much is now understood and rich detail has been revealed, but the problem remains fascinating, in that a final resolution can hardly be said to have been reached. What might have seemed a simple matter has proved to be deep and wide, causing a re-examination of foam rheology at a fundamental level. There are no further published results from the Debrégeas instrument, but a wealth of further data has emerged from other groups, as reviewed in section 3 .

Some of the main theories that are currently advanced for localization and the underlying dynamical theory arise out of the work of the Dublin [23, 24, 25, 26, 27] and Leiden [28, 29, 30] groups. This body of work, based on a continuum approximation, is not yet reconciled with alternative descriptions based an quasistatic simulations by Kabla et al. [31, 32, 33] and the Aberystwyth group [34, 35, 36]. Our first objective is to put the contributions of the Dublin and Leiden groups in the simplest complete and coherent form, as a basis for continued debate. We will however adduce some detailed simulations, in which the individual bubbles are represented, in an attempt to better understand the continuum model.

We begin by comparing 2D and 3D foams in order to introduce wall drag, the force that resists motion relative to a confining plate. We will later see that the 

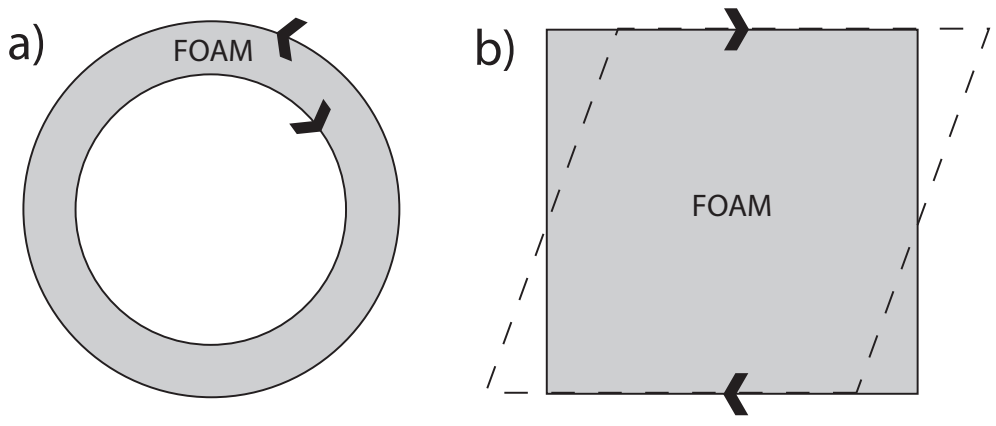

Figure 3. The two types of experimental setups under consideration: (a) a circular (Couette) geometry, and (b) a straight geometry. The arrows indicate the shearing boundaries which may be displaced during an experiment or computer simulation to induce shear.

introduction of wall drag has the general consequence of shear localization, with various forms and dependencies, according to the particular case. It is therefore a crucial factor in interpreting shear localization experiments.

We will also briefly review the experimental comparisons that can be made. They largely validate the theoretical model, but additional confirmation will surely be needed, as well as further refinements of the theory.

Throughout the review we will mainly use the term shear localization, rather than shear banding. The latter term is familiar in materials science, but usually refers to localized bands of shear within the sample, whereas in the present case it is to be found only at a boundary. This distinction may be a mere matter of taste, but we prefer to avoid the connotations of the alternative expression. A more general and in many respects complementary review of "Shear Bands in Matter with Granularity" has been recently compiled by Schall and van Hecke [37].

A further type of localized deformation is possible in solid foams. Here, compression might lead to the collapse of a cell which either has a lower collapse stress or is subject to a locally higher stress [?]. This cell collapse might then propagate to cells in its vicinity, resulting in a compaction band where the cellular structure has an increased local density. Since this review article concerns liquid foams only we will not discuss such bands. In all the experiments described below the gas in the bubbles can be treated as incompressible.

In planning our article we faced what might be called "the reviewer's dilemma". 
If a subject is to be reviewed in the midst of its progress, all sorts of loose ends, untested assumptions and questionable generalizations will be found. On the other hand, if we wait until all is clear, it may have become stale and uninteresting! Certainly the present review falls into the "work in progress" category, and criticism will be very welcome.

We shall first review all of the main experiments and their essential results, before embarking on theoretical interpretations.

\section{The experiment of Debrégeas, Tabuteau and di Meglio}

In 2001 Debrégeas et al. noted that whereas localized shear in granular material and soil mechanics "has recently received a lot of attention from physicists [...] a clear picture has not emerged yet." [22] They chose to work on 2D foams in the belief that "foams may shed light on the dynamics of granular systems by evidencing the minimal set of ingredients needed to get shear banding" [22]. The relationship of what is reviewed here to corresponding studies of granular systems is indeed a fascinating topic. We will comment on it only very briefly in section 16 .

The apparatus consisted of a 2D circular Couette rheometer in which foam was confined between two glass plates (as in Figure 2(c)). The outer to inner radius ratio was $122 \mathrm{~mm} / 71 \mathrm{~mm}$, but note that the shear band that was eventually measured had a width of only a few millimetres. This is why the experiment can be considered equivalent to one in which simple shear is imposed and the circular geometry (which leads to a $r^{-2}$ stress decay) can often be ignored, as is the case here.

The bidisperse foam was confined between plates with a separation of $2 \mathrm{~mm}$, and the resulting $2 \mathrm{D}$ bubbles had diameters only slightly greater than this (bubble diameter $2.0 \pm 0.2 \mathrm{~mm}$ and $2.7 \pm 0.2 \mathrm{~mm}$ respectively).

The inner circle was a movable wheel, which was rotated to impose a steady velocity of up to $V=0.7 \mathrm{~mm} / \mathrm{s}$ at the boundary of the foam. It was reported that within this range "the velocity profiles were similar apart from an overall scale factor" [22], presumably meaning that the profile did not change apart from being proportional to the boundary velocity. This is an important comment: later contributors were to point to significant velocity-dependence in their respective data, and we shall return to this matter in due course. The actual data that was reported for the original experiment was for $V=0.25 \mathrm{~mm} / \mathrm{s}$ ( Debrégeas et al. referred to this as the quasistatic regime). Accordingly the shear rates in the steady state were 


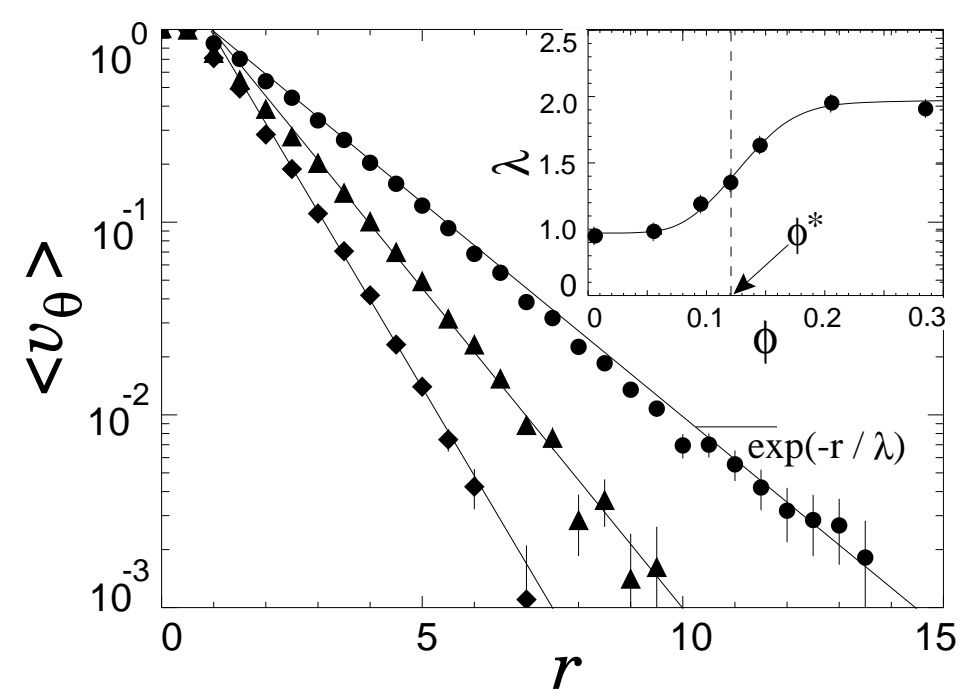

Figure 4. Measured velocity distributions in the original experiment by Debrégeas et al. for Couette geometry [22]. The three different symbols mark datasets obtained for different liquid fraction. The inset shows the variation of the localization length with liquid fraction. Reprinted figure with permission from G. Debrégeas [22]. Copyright 2001 by the American Physical Society.

of the order of $0.1 \mathrm{~s}^{-1}$ within the shear band, since its width was of order of a few millimetres.

Video sequences, which were kindly made available to other groups $\ddagger$, showed the process by which localization of shearing motion developed at the inner boundary, but in taking data this transient regime was avoided by allowing a full rotation before making measurements. This initial preparation corresponded to a nominal shear of the whole sample, the magnitude of which was roughly 5 , and an even greater value within the region of localized shearing motion that becomes established during the rotation.

The velocity profile associated with shear localization at the moving inner boundary was consistent with an exponential form (over three orders of magnitude), that is

$$
v(x) \propto \exp (-x / l),
$$

$\ddagger$ http://tel.archives-ouvertes.fr/docs/00/04/56/98/HTML/Manip.mpg - We thank A. Kabla for this link. 
as shown in figure 4. Here $l$ (represented by $\lambda$ in the notation of these authors) is the localization length and is the main object of qualitative and quantitative scrutiny in most of the present article, although it will not always be associated with an exponential profile.

Debrégeas et al. went on to present the variation of $l$ with liquid fraction $\phi$ of the foam. The liquid fraction is difficult to estimate or even define satisfactorily in the present context [38]; we shall not take up this aspect of the results. Correlations of bubble motions were also analyzed.

Setting these matters aside, we may summarise some basic findings of the paper as follows.

- There is localization at the moving boundary.

- The velocity profile has an exponential form.

- The localization length shows no variation with boundary velocity, within the range of velocities that was used.

\section{Later experiments}

To our knowledge, no further data has yet been reported based on the use of the particular apparatus described in the last section. Instead, various other set-ups have been employed for the same purpose, namely by the groups of Dennin and van Hecke at the universities of California-Irvine and Leiden, respectively. Here we will note the nature of these experimental variations, together with some of the key findings. A more detailed interpretation of the data is left to later sections, when its implications will be clearer.

Changes in sample geometry (from Couette to simple shear), boundary conditions, and other technical details are not generally important, but the change of foam type (among the three options of figure 2) certainly is.

\subsection{Experiments by Dennin's group using a straight geometry}

For a straight geometry the group of Dennin found that whereas shear is not localized in the case of a Bragg raft (Figure 2(a)), strong localization at the moving boundary occurs when the raft is covered by a glass plate (Figure 2(b)) [39]. In this second case the localization length was found to be approximately three average bubble 
diameters, independent of the velocity of the moving boundaries. (Since the applied strain rates were $0.0014,0.0028$ and $0.014 \mathrm{~s}^{-1}$ one can deduce local strain rates between 0.005 to $0.05 \mathrm{~s}^{-1}$ for the sheared regions.)

The foam samples in the above experiments were nearly monodisperse (bubble diameters in the range $2.43 \pm 0.08 \mathrm{~mm}$ ) and the photo of a sample shows that the bubbles crystallize in various domains (see figure 3 of [39]). This appears to be the main contributing factor for the observed velocity independence of localization length in the sheared covered bubble raft. A similar behaviour for monodisperse foams was found in the experiments of the Leiden group ([28] and Section 3.3) and also in dynamic computer simulations of the viscous froth model (Section 12.3).

\subsection{Experiments by Dennin's group using Couette geometry}

Dennin's group performed further series of experiments using a Couette geometry with moving outer cylinder and bubbles in covered [43] and uncovered Bragg rafts [44, 41, 43] (Figure 2(b) and 2(c) respectively). Here the range of bubble diameter was larger than in the above experiments [39] and the issue of crystallisation should thus not arise.

Of particular relevance to the continuum theory of localization (and indeed in part inspired by this theory) are the experiments carried out in 2008 for both bubble raft and covered bubble raft in a Couette geometry with a radius ratio of $80 \mathrm{~mm} / 22.5 \mathrm{~mm}$ [43]. For the bubble raft it was found that shear localizes at the inner boundary as the outer boundary was rotated. The localization length was approximately $3.5 \mathrm{~mm}$, independent of rotation velocity. For the covered bubble raft the localization length was found to increase with boundary velocity, from about $3 \mathrm{~mm}$ to about $4.5 \mathrm{~mm}$ (These widths were extracted from fits of the velocity profiles to exponentials.) Increasing the boundary velocity eventually led to the formation of a second shear band at the outer (moving) boundary. Its width was found to decrease with velocity, from about $16 \mathrm{~mm}$ to $6 \mathrm{~mm}$.

The occurrence of a second shear band had been predicted by Clancy et al. [24] from numerical solutions of the continuum model in circular geometry. The possibility of a shear band at the outer moving cylinder was also demonstrated by the dynamic viscous froth simulations of Cox for sufficiently high shear rates [34]: for lower rates, and also in quasi-static simulations, he found that localization occurs at the inner, non-moving boundary. 


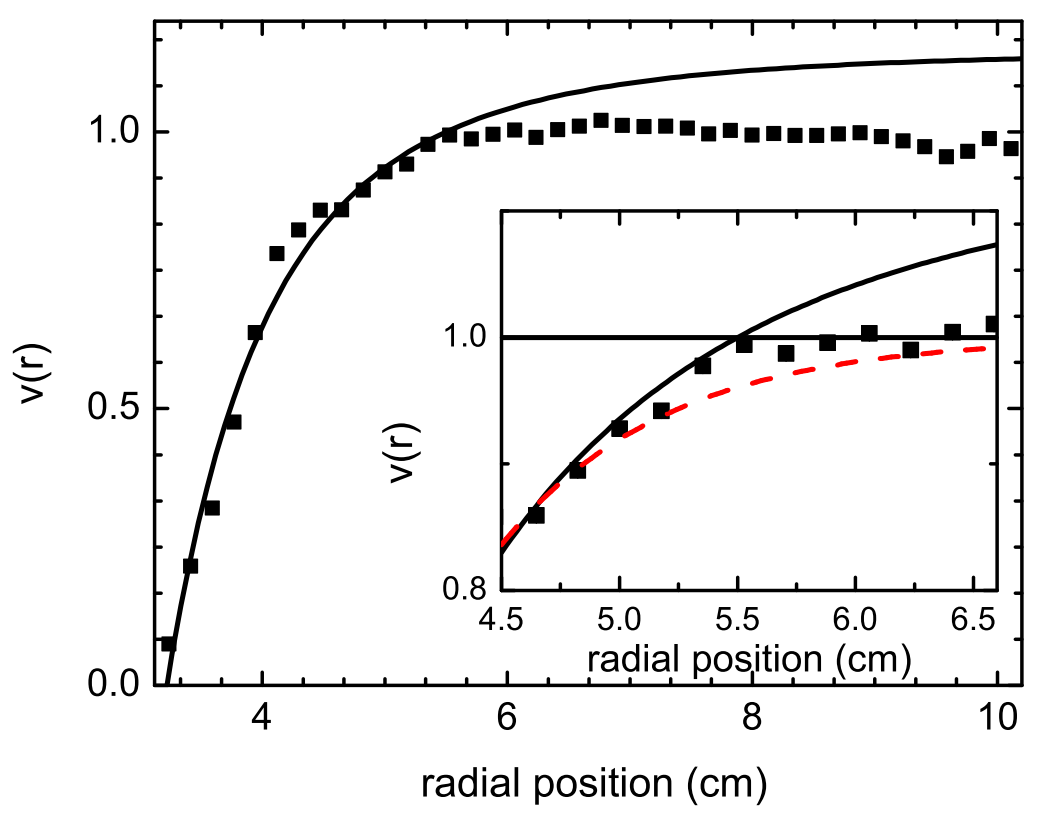

Figure 5. Data from the Dennin group showing shear localization in the case of circular (Couette) geometry (reprinted figure 5 from [40]). The scaled azimuthal steady-state velocity $v(r) /(r \Omega)$, where $r$ is the radial position and $\Omega$ is the rotation rate of the outer cylinder, may become constant at some internal point. In the data of $[41,42,40]$, taken for a moving outer boundary and a fixed inner one, there is a discontinuity of $d(v(r) /(r \Omega)) / d r$ at this point. Note that this published figure uses the symbol $v(r)$ to denote $v(r) /(r \Omega)$ in the notation of the present paper. The solid and dashed lines show least-square fits to power-law and exponential, respectively. This figure is used with permission from M. Dennin [40], Copyright 2008 by IOP Publishing Ltd.

The full scenario for Couette geometry is rather rich [24], but in terms of physical interpretation it adds little to the simple shear experiments upon which we concentrate in this review. But one feature of the results, associated with the edge of the rigidly rotating region, is intriguing. The velocity profiles in Dennin's experiments on bubble rafts in Couette geometry show that the derivative of the profile with respect to radial position is discontinuous at the radial position that separates rigid body motion from shear flow [41, 42, 40]; see for example Figure 5. We will discuss the implications of this for a refinement of the continuum theory in 
section 10 .

\subsection{Experiments of the Leiden group}

In yet another variation, the group at Leiden experimented with a bidisperse bubble monolayer (equal number of bubbles with diameter 1.8 and $2.7 \mathrm{~mm}$ respectively) on top of a liquid pool and covered by a glass plate (2D foam type b) of Figure 2) [28]. The distance between liquid surface and glass plate was $2.25 \mathrm{~mm}$. The geometry of the set-up was straight; parallel boundaries where moved at constant speed in opposite directions by two counter-rotating acrylic glass wheels, placed perpendicular to the plane of the bubble monolayer. Six different velocities were used, ranging between 0.026 and $8.4 \mathrm{~mm} / \mathrm{s}$. Shear localization at the two boundaries was found to increase with velocity, as is evident in Figure 6. From the data shown one can estimate the range of applied shear rate in the respective shear bands as roughly $10^{-3}$ to $0.5 \mathrm{~s}^{-1}$.

The variation of the localization length with boundary velocity was not explicitly shown in the original publication [28]. It has since been computed by M.E. Möbius
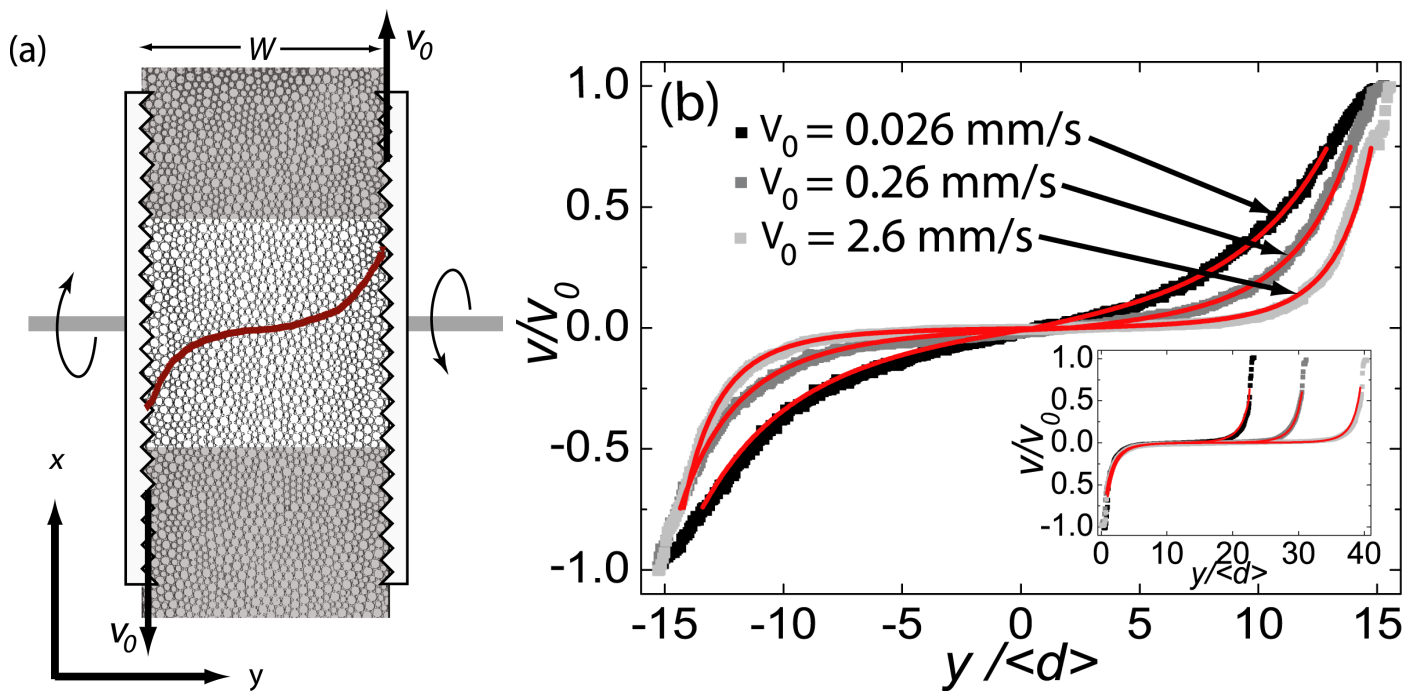

Figure 6. The Leiden experiment [28]. (a) A monolayer of foam is confined between a liquid pool and a covering glass plate. (b) Localization takes place close to the moving boundaries, the localization length decreases with boundary velocity. Reprinted figures with permission from M. van Hecke and [28]. Copyright 2008 by the American Physical Society. 


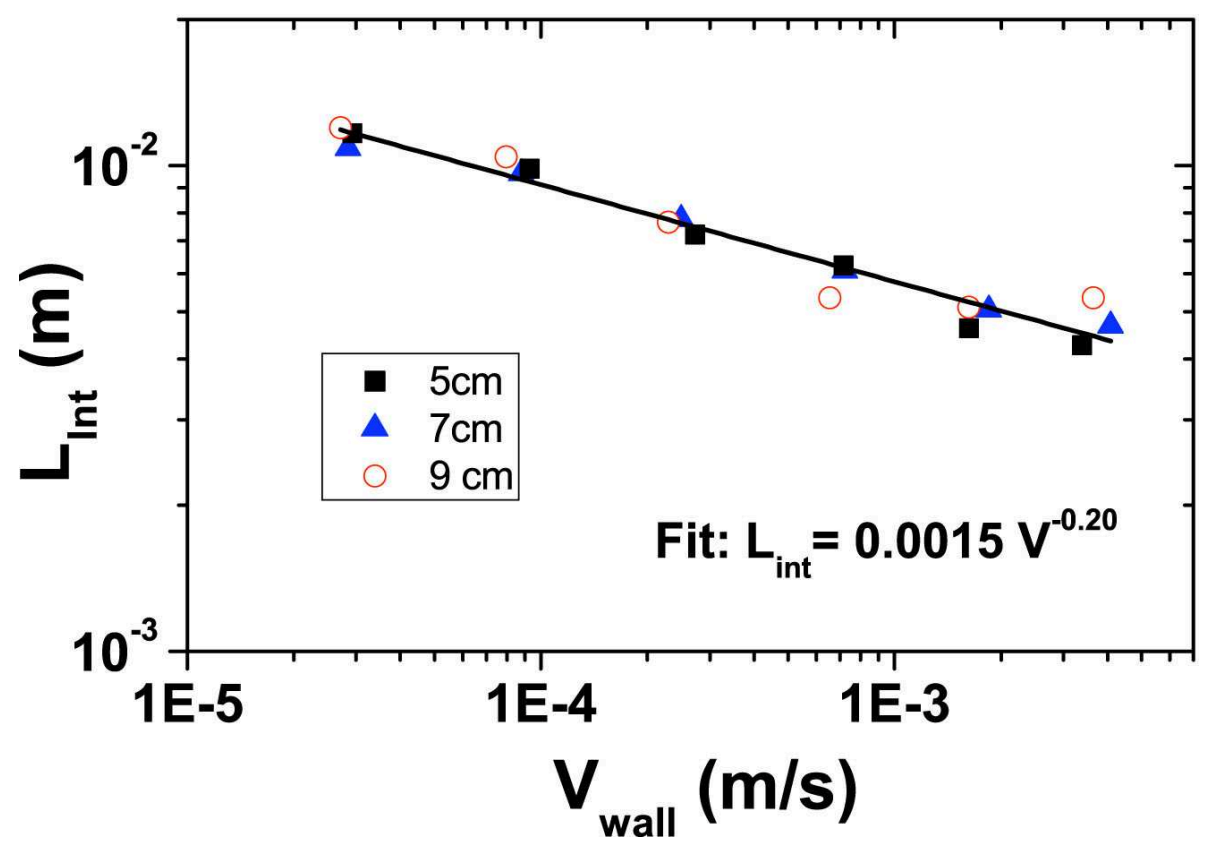

Figure 7. Variation of the localization length $l_{\text {int }}$ with wall velocity $V$ in the Leiden experiments on bi-disperse foams [28]. The data is well described by a power law, $l_{\text {int }} \propto V^{-0.20}$, where $l_{\text {int }}$ is computed from a numerical integration of the measured velocity profiles, see Equation (26). The different symbols correspond to different values for the distance between the counter-rotating wheels. This figure was kindly provided by M.E. Möbius of the Leiden group.

and is shown in Figure 7. The localization length decreases with wall velocity in the form of a power law with exponent -0.2. We will interpret this value in section 8 in terms of the continuum model.

It is noteworthy that no such velocity dependence was found in the experiments of the Leiden group when using monodisperse bubbles, which are more or less ordered, in contrast to the disordered arrangements of bidisperse or polydisperse samples. In this case the localization length was between one and two bubble diameters, independent of the shear rate. This matches the result of Dennin's group [39] for similar experimental conditions. 


\subsection{Summary of experimental results}

Table 1 summarises the experiments and their essential results, as regards localization. As we have seen, experimental variations include the type of $2 \mathrm{D}$ foam (Figure 2) and the distribution of bubble sizes (monodisperse, bidisperse, polydisperse), the geometry of the experiment (straight or Couette geometry) and the shear rates used.

SUMMARY OF EXPERIMENTS ON SHEAR LOCALIZATION

Hele-Shaw (Wall Drag)

\begin{tabular}{|c|c|c|c|c|}
\hline Geometry & Ref & Localization & At Boundary & Distribution \\
\hline Circular & {$[22]$} & yes & inner moving & bidisperse \\
\hline \multicolumn{5}{|c|}{ Confined Bragg Raft (Wall Drag) } \\
\hline Geometry & Ref & Localization & At Boundary & Distribution \\
\hline \multirow{3}{*}{ Straight } & {$[39]$} & yes & moving & monodisperse \\
\hline & {$[28]$} & yes & moving & monodisperse \\
\hline & [28] & yes* & moving & bidisperse \\
\hline Circular & {$[43]$} & yes* & inner stationary, outer moving & polydisperse \\
\hline \multicolumn{5}{|c|}{ Bragg Raft (No Wall Drag) } \\
\hline Geometry & Ref & Localization & At Boundary & Distribution \\
\hline Straight & {$[39]$} & no & - & monodisperse \\
\hline \multirow{4}{*}{ Circular } & {$[44]$} & no & - & tridisperse \\
\hline & {$[41]$} & yes & inner stationary & polydisperse \\
\hline & {$[42]$} & yes & inner stationary & polydisperse \\
\hline & {$[43]$} & yes & inner stationary & polydisperse \\
\hline
\end{tabular}

Table 1. Relevant detailed experiments of two-dimensional foam under shear. Categorization is by geometry and experimental setup. Experiments where localization length is found to depend on boundary velocity are marked with an asterix $(*)$. 


\section{Towards a theory: are $2 \mathrm{D}$ foam properties really similar to those of $3 \mathrm{D}$ foam?}

We begin theoretical deliberations with the above question. When the answer is yes, this offers us one of the great simplifications of the qualitative and semi-quantitative physics of foams, extending to such subjects as structure, coarsening, elasticity and plasticity [6]. It has become almost an article of faith, but has proved misleading for rheology.

The point is simply that in most cases (including that of the seminal experiment of the Debrégeas group [22]), the $2 D$ foam is in contact with a solid plate. This does not matter much for static properties, but when the foam flows it entails a resisting force at the surface, which we shall here call wall drag. We shall argue that this can play a primary role in causing shear localization.

Wall drag has absolutely no counterpart in the bulk rheology of 3D foam, so it injects an essential difference into the $2 \mathrm{D}$ case, whenever it is present. It cannot be relevant to shear localization in three dimensions, which is sometimes seen. We shall have very little to say on that matter in the present article.

Wall drag is, in our view, essential to the analysis of shear localization in 2D, in general. There are therefore two essential ingredients to be combined in a theory: some description of the internal forces or local rheological response of the foam, and the external drag force whenever this is present. It is absent, at least to a first approximation, in the experimental $2 \mathrm{D}$ system of figure $2 \mathrm{a}$ ), but present in the other $2 \mathrm{D}$ foam types.

In the elementary continuum theory, it turns out that the localization length is determined by a competition between the internal dissipative forces (which tend to delocalize shear) and the external ones (which tend to enhance localization). The main theme of this article is the continuum theory that expresses this competition. By its nature it cannot capture all of the physics but, in as much as it proves to be valid, it illuminates the subject in simple terms, and makes definite predictions.

Mention should be made of an important development in the rheology of 3D foams, which may well have a bearing in the context of the present review, but this remains to be worked out and confirmed. Goyon et al. [45] have interpreted a range of rheological experiments on different length scales as conclusively demonstrating nonlocal effects in the relationship between stress and strain rate. In these experiments the extent of the nonlocality is considerable, extending to five or more 
bubble diameters. These are 3D foam experiments, but there seems no reason why their implications should not extend to 2D foams as well.

\section{Simulations showing shear localization}

The complementary approach of detailed simulation instead of a continuum description has played an important role from the outset. In this, the detailed motion of the soap films (or other elements representative of 2D foam structure) are followed. In such a simulation the role of the local rearrangements ( $\mathrm{T} 1$ processes) of cells can be analyzed, whereas it is buried in the empirical parameters of the continuum model.

The first theoretical analysis of the Debrégeas experiment was based on such a 2D simulation [31] in which a 2D foam was fully represented by lines (cell walls), vertices, cell pressures etc., in a tradition dating back (at least) to the $1980 \mathrm{~s}$ $[46,47,48]$. Two key aspects distinguish that approach from the main one considered in this article. Firstly these simulations (and various later ones [32, 33, 35]) were quasistatic, that is, they proceeded by a sequence of small changes in the boundary conditions (to represent an imposed shear), equilibrating the structure at every step. No finite shear rate is defined. Secondly, they did not include wall drag.

It follows that in a quasistatic simulation, Galileian invariance applies: precisely the same procedure should be applicable to the natural dynamics of the system in any reference frame. In simple shear, the imposition of a velocity $V$ at one top boundary should be entirely equivalent to the imposition of velocity $-V$ at the opposite boundary (with zero velocity at the other boundary in each case). If localization is to be found, it ought to occur at either boundary with equal probability. This does not appear to occur in the reported results. However, in the quasistatic simulations of REF THESIS WYN, up to an applied strain of 5, the localized region is seen to slowly move between the boundaries, maintaining a roughly constant width. This might be indicative that in the quasistatic case localization is possible at both boundaries.

It is possible to introduce wall drag, by use of the $2 \mathrm{D}$ viscous froth model $[49,50]$ (see section 12), or the soft disk model [27] (see section 13), but it cannot play any role in a quasistatic simulation. Any such simulation (or continuum model) that includes a wall drag has a preferred frame of reference (that in which the confining plate is stationary) and Galilean invariance does not apply. Indeed we would insist that in such cases, simple shear must lead to localization at the moving boundary, for the case of a straight geometry. 
SUMMARY OF SIMULATIONS ON SHEAR LOCALIZATION

With Wall Drag

\begin{tabular}{cccccc}
\hline Geometry & Ref & Model & Localization & At Boundary & Distribution \\
\hline \multirow{4}{*}{ Straight } & {$[27]$} & Soft Disk & yes & moving & monodisperse \\
& {$[27]$} & Soft Disk & yes* & moving & polydisperse \\
& {$[50]$} & Viscous Froth & yes & moving & monodisperse \\
& {$[50]$} & Viscous Froth & yes* & moving & polydisperse \\
\hline Circular & {$[34]$} & Viscous Froth & yes & outer moving & polydisperse \\
\hline
\end{tabular}

\section{Without Wall Drag}

\begin{tabular}{cccccc}
\hline Geometry & Ref & Model & Localization & At Boundary & Distribution \\
\hline \multirow{5}{*}{ Straight } & {$[27]$} & Soft Disk & no & - & polydisperse \\
& {$[31]$} & Quasistatic & yes & moving, stationary & polydisperse \\
& {$[33]$} & Quasistatic & yes & moving, stationary & polydisperse \\
& {$[35]$} & Quasistatic & yes & moving, stationary, bulk & polydisperse \\
\hline Circular & {$[34]$} & Quasistatic & yes & inner stationary & polydisperse \\
\hline
\end{tabular}

Table 2. Relevant detailed simulations of two-dimensional foam under shear. Categorization is by geometry and the presence or absence of wall drag. Simulations where localization length is found to depend on boundary velocity are marked with an asterix $(*)$. Note also that many of the much earlier $(1980 \mathrm{~s}$ and $90 \mathrm{~s})$ simulations applied only extensional shear to the sample [48, 51, 52, 53, 54], rather than the simple shear for which localization has been observed.

In Table 2 we list relevant features and results for a variety of different detailed simulations. Viscous froth and soft disk model will be described in sections 12 and 13, respectively, and the results of these simulations will be interpreted in terms of the continuum model which we now develop.

\section{Continuum theory}

That localization was found at all in the quasistatic simulation of simple shear [31] will initially constitute a paradox from the point of view of the present article (since there is necessarily no role for wall drag in such a simulation and we cannot attribute 
localization to this cause). We will not attempt to resolve it until section 10 . For the meantime we pursue the course of continuum theory with wall drag, as outlined already. In this we deal with local averages of stress, strain, strain rate and related quantities, rather than the description of individual bubbles or cells. The boundary velocity $V$ is a key parameter, in contrast to quasistatic treatments whose results are, by their very nature, $V$-independent.

This smoothed average picture must be questioned whenever the localization length becomes so small as to be comparable with bubble size, or according to the recent ideas of Goyon et al. [45], the range of nonlocal effects. But it seems to have a wide range of validity and is, of course, a great simplification.

\subsection{Wall drag}

In such a picture, the local drag force at any point $\vec{r}$ in the moving foam contributes a body force (per unit area) $\vec{F}_{d}(\vec{r})$ which is taken to be a function of local average velocity $\vec{v}(\vec{r})$ :

$$
\vec{F}_{d}(\vec{r})=-c_{d}|\vec{v}(\vec{r})|^{b} \frac{\vec{v}(\vec{r})}{|\vec{v}(\vec{r})|},
$$

with a positive drag force coefficient $c_{d}$. That is, the magnitude of the drag force is proportional to the local speed raised to the power $b$, and acts in direct opposition to the local velocity of the foam.

Given a power law for the drag force on one of the lines (cell walls) of Figure 1, it is obvious enough how to average it to obtain such a formula; in particular, the index $b$ is the same for the local force and the average. This index is not found to be unity as one might expect, and usually takes much lower values [55, 19], as explained in Section 15.

Since the average bubble motion is fixed by the direction of applied shear, we will in the following use the scalar form for the drag force (per unit area), which may be written (for positive velocity v, in the y direction, as in Figure 10 ) as

$$
F_{d}(x)=-c_{d} v(x)^{b} .
$$

This is the first and crucial ingredient of the continuum theory. 


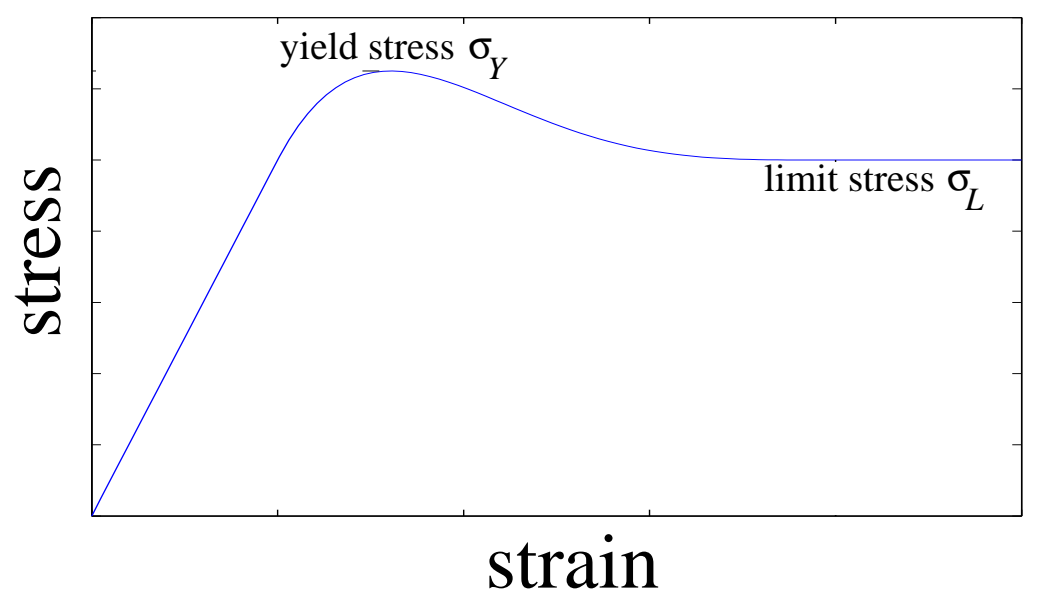

Figure 8. Quasi-static stress-strain relation with distinct yield and limit stresses, for steadily increasing strain.

\subsection{Constitutive relation}

The second ingredient is a local constitutive relation, relating averaged local stress, strain and strain rate. It is a traditional concept, but entails many questions and difficulties, in general.

First let us remind ourselves of the local response of a foam (3D or 2D) to slowly and steadily increasing shear ("quasistatic conditions"). It is sketched in figure 8.

For low stress or strain the foam is a linear elastic medium. In the opposite limit, as strain goes to infinity, the stress is constant. In what follows, the system will often be in the latter regime, tending to an eventual steady state in which the system is continuously sheared. Usually we shall not be concerned with the earlier transient regime.

In the model that we are developing, it is often convenient to neglect small differences between yield stress $S_{Y}$ and limit stress $S_{L}$ indicated in figure 8, which are in practice of the order of ten percent of $S_{Y}[?, 50]$. Considerable simplification is achieved in replacing the curve by a monotonic dependence of stress on (steady) strain, as in figure 9. Adopting this approach, the symbol $S_{Y}$ stands for both yield and limit stress, treated as identical, at least for now.

All this refers to the imposition of quasistatic steady strain. At finite strain rates an additional stress is entailed, which we may loosely term the "viscous" stress 


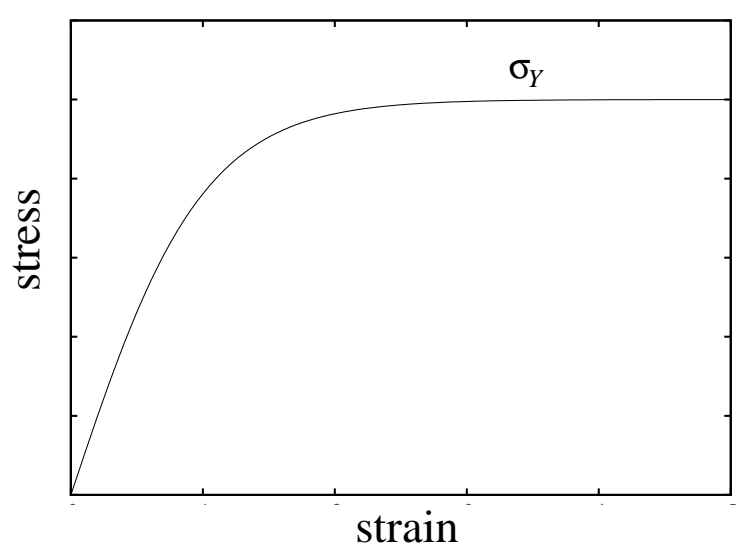

Figure 9. Simplified model form (expressed by the function $f$ in the text) for a monotonic quasistatic stress-strain relation, for steadily increasing strain. No distinction is made between yield and limit stress. The functional form illustrated is that of the $\tanh (x)$ function.

for this. It increases with strain rate, but not usually in a simple linear form.

This additional stress is captured in the empirical Herschel-Bulkley relation. It is familiar in the long history of rheometry applied to 3D foams, much of it driven by industrial applications. The analysis of experimental rheological data for foams and analogous systems has been highly empirical, and has usually rested on the formalism of this relation [56]. It is just a power-law dependence of the excess stress $S-S_{Y}$ on strain rate $\dot{\gamma}$, for the steady shear that occurs above the yield stress $S_{Y}$,

$$
S=S_{Y}+c_{v} \dot{\gamma}^{a}
$$

Here $c_{v}$ is the coefficient of the viscous contribution to stress, also called consistency.

Often in the present article, shear is generally taken to denote local simple shear (for the somewhat subtle distinction between simple and extensional shear see [?]). The experiments cited all involve local simple shear but the overall macroscopic geometry of the rheometer may be straight (see Figure 3b)) or circular (Couette, see Figure 3a)).

If the Herschel-Bulkley exponent $a$ is unity, Equation (4) may be called the Bingham relation [56]. This is rarely observed but may be useful for qualitative considerations. Instead the exponent generally takes a value significantly less than unity. 
The experiments with which we are concerned mostly involve the imposition of steady shear in one sense only. For this case one may choose to replace Equation (4) by the more general relation

$$
S=S_{Y} f\left(\gamma / \gamma_{Y}\right)+c_{v} \dot{\gamma}^{a}
$$

which is not confined to the steady shear state into which the system eventually settles, under an imposed strain $\gamma>\gamma_{Y}$ ( $\gamma$ denotes strain and $\gamma_{Y}$ yield strain). The first term on the right hand side represents the dependence sketched in Figure 8 or Figure 9. This relation can be used for any imposed stress (usually constant here), such that $\dot{\gamma}>0$. That is, the direction of shear cannot be reversed without modification of Equation (5).

At low strain $\gamma$, the function $f\left(\gamma / \gamma_{Y}\right)$ increases linearly with strain and represents the (scaled) elastic stress in the linear elastic regime. In much of the work reviewed here, the function $f$ was given a specific form such as that of the hyperbolic tangent (Figure 9).

Eventually we will have cause to revisit this simplification in search of the effects of the peak in Figure 8.

An obvious question is: how can we account for the observed values of $a$ ? Does it reflect a corresponding dependence of local forces on local velocities? Or does it have some deeper significance? Such questions were raised in an earlier brief review [57], and part of the justification for the present one is the availability of some answers, but we will delay this discussion until section 14 .

To summarise, our initial viewpoint is very restrictive and may be contrasted with that of theories that seek to develop a theory for any arbitrarily varying imposed stress as a function of time, and more complicated geometries. Ultimately, such generality will be essential, but in the specific debate over localization it is probably unhelpful, since it would bring in an unwieldy mass of mathematical formalism.

\section{Detailed results for the continuum model}

The properties and predictions of the continuum model have been analyzed in a series of papers $[23,24,25,26,28,30,58]$. Its main implications may be summarized very straightforwardly, provided that we adhere to the restrictions already suggested, and in particular: 
- confine attention for the present to the case in which simple shear is imposed, rather than the circular or cylindrical geometry of the original experiment [22], for which it was often justifiably claimed that the geometry had little effect. We refer to this circular case as "Couette" geometry.

- set aside for the moment the detailed discussion of transient effects, that is, the evolution of motion towards the eventual steady state.

- make further approximations consistent with strong localization, that is, localization with a scale much less than that of the sample geometry. This means that the immobile boundary plays little role when the other is set in motion.

None of these simplifications are strictly required to progress the analysis but together they reduce the mathematics to a minimum, with no distracting diversions.

We consider a steady state of simple shear, with an average profile $v(x)$, as in figure 10, and will quickly show that it is localized, as illustrated.

\subsection{The continuum model equation}

The $2 \mathrm{D}$ foam is sheared by the imposition of motion at one boundary at $x=0$ with velocity $V$, while at the other, at $x=L$, it is stationary (and for present arguments $L$ will be taken to infinity). The local strain rate is given by $\dot{\gamma}=|d v(x) / d x|$.

Neglecting inertia and hence equating the total force on an element to zero (as sketched in figure 11), the balance of the two forces on the element (arising from the Herschel-Bulkley relation (Equation (5)) and wall drag (Equation (3)), results in

$$
S_{Y} \frac{d}{d x} f\left(\gamma / \gamma_{Y}\right)+c_{v} \frac{d}{d x}\left|\frac{d(v(x))}{d x}\right|^{a}=-c_{d}(v(x))^{b} .
$$

Restricting ourselves to a discussion of the steady state at long times, the above equation is reduced to

$$
\frac{c_{v}}{c_{d}} \frac{d}{d x}\left|\frac{d v(x)}{d x}\right|^{a}=-v(x)^{b} .
$$

We will now introduce dimensionless velocities $\hat{v}(x)$, defined by

$$
\hat{v}(x)=\frac{v(x)}{V}
$$




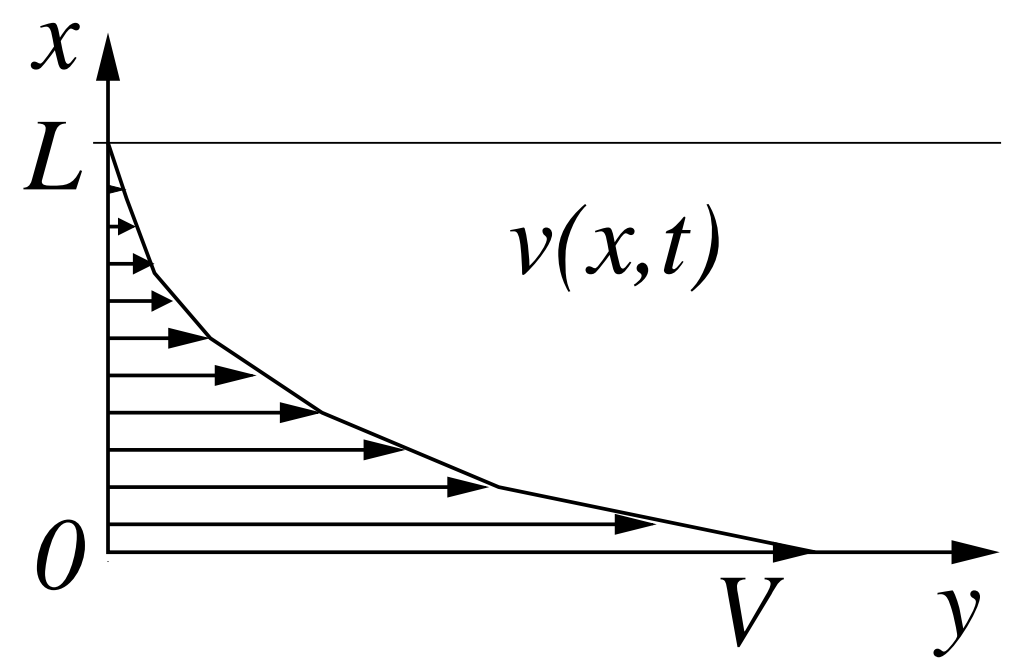

Figure 10. Sketch of velocity profile $v(x)$ for a straight geometry (see Figure $3 \mathrm{~b})$ ). The foam is moved at the boundary $x=0$ with velocity $V$ and is stationary at the boundary $x=L$.

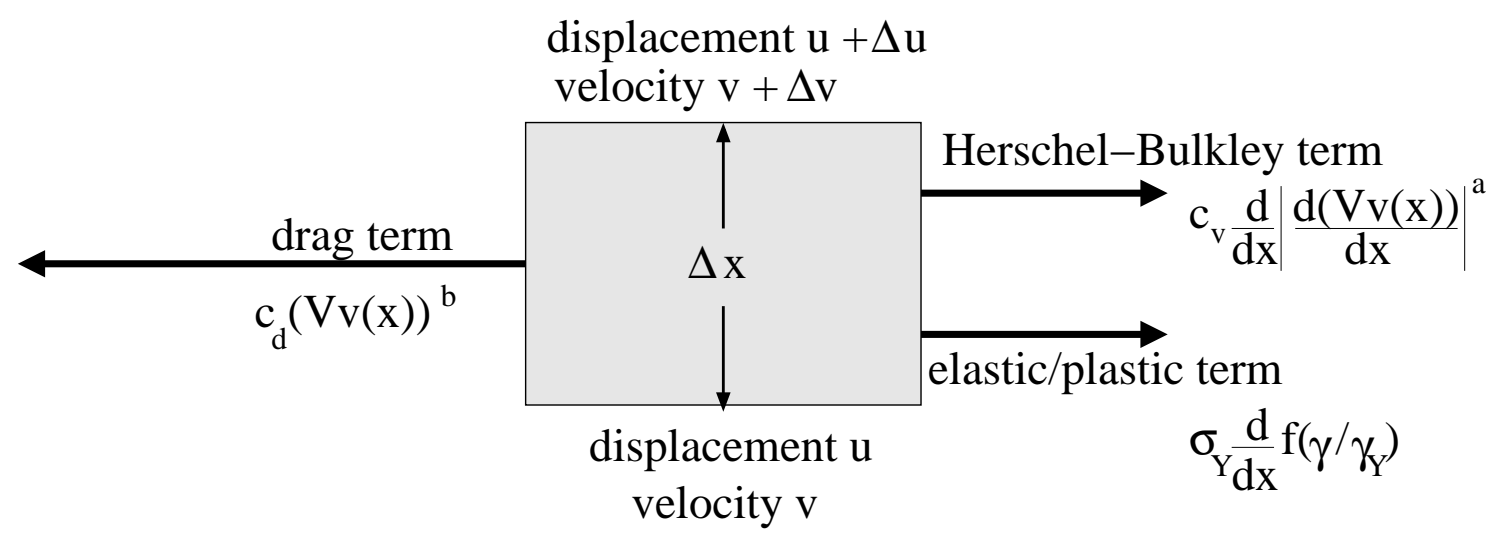

Figure 11. Forces per area acting on an element of a $2 \mathrm{D}$ foam under shear. (Note that in 2D stress has dimensions of force per length.) 
leading to the boundary condition $\hat{v}(0)=1$. Thus Equation $(7)$ is reduced to

$$
k \frac{d}{d x}\left|\frac{d \hat{v}(x)}{d x}\right|^{a}=-\hat{v}(x)^{b}
$$

where the (positive) parameter $k$ is given by

$$
k=\frac{c_{v}}{c_{d}} V^{a-b} .
$$

It contains the only dependence of the (scaled) equation on the velocity $V$ of the moving boundary. In the following we shall proceed to solve for the steady-state profile $\hat{v}(x)$ that is our concern.

Equation (9) relies on the assumption (consistent with the eventual solution given below), that everywhere $\frac{d \hat{v}}{d x}$ is negative and $\hat{v}$ is positive. This avoids the complications of hysteresis when $\frac{d \hat{v}}{d x}$ is reversed [59].

\subsection{Solutions of the continuum model equation: velocity profiles}

Often Equation (9) is to be solved under the boundary conditions

$$
\hat{v}(0)=1 \text { and } \hat{v}(L)=0 .
$$

However, if the profile is localized on a scale $l$ much less than $L$, we may judiciously take the second condition to apply at infinity, so that

$$
\lim _{x \rightarrow \infty} \hat{v}(x)=0
$$

replaces the second part of the boundary condition (Equation (11)).

Equation (9) then has a trivial solution that may be obtained by the traditional methods applied to differential equations, or simply by inspection or trial solution, as follows. Finding the solution is complicated only by the existence of three distinct cases. (Recall that $a$ and $b$ are model parameters, as yet unspecified, although both are positive. Their values determine the type of solution to be applied.)

It is immediately evident that, in general, a power-law dependence of $\hat{v}$ upon $x$ may offer a solution of Equation (9).

The ansatz

$$
\hat{v}(x)=\left(1-x / x_{0}\right)^{n},
$$

satisfies the boundary condition $\hat{v}(x=0)=1$. 


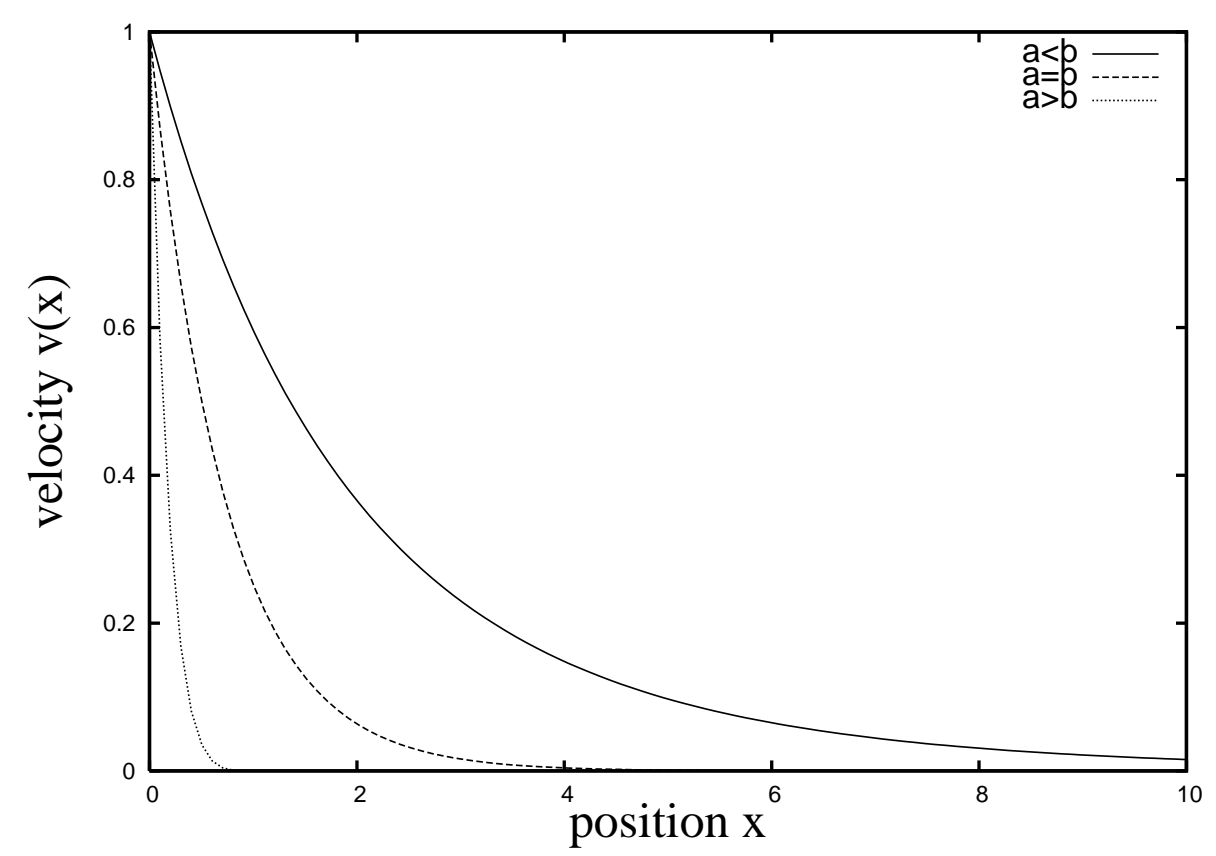

Figure 12. Velocity profiles illustrating the three distinct cases $a<b$ (power law), $a>b$ (truncated power law) and $a=b$ (exponential), where $a$ and $b$ are the respective exponents in Herschel-Bulkely and viscous drag relation.

For the case $x_{0}<0$ it is acceptable for all $x$. We consider this case first. Substitution into Equation (9) shows that the ansatz is indeed a solution, with the exponent $n$ given by

$$
n=\frac{1+a}{a-b}
$$

The value of $x_{0}$ needs to be determined by equating prefactors, giving

$$
\frac{a(n-1)}{x_{0}} k\left|\frac{n}{x_{0}}\right|^{a}=1 \text {. }
$$

Since $x_{0}<0$ this requires $n<1$, i.e. $a<b$. The value of $x_{0}$ is given by

$$
x_{0}=\frac{1+a}{a-b}\left(\frac{a(1+b)}{1+a} k\right)^{\frac{1}{1+a}} .
$$

In summary, for $a<b$, there is a power law solution for $\hat{v}(x)$ which decreases continuously as $x$ tends to infinity. An example of such a profile is shown in Figure 12. 
For $x_{0}>0$, the solution of Equation (9) may be developed as

$$
\hat{v}(x)= \begin{cases}\left(1-x / x_{0}\right)^{n} & \text { for } x \leq x_{0} \\ 0 & \text { for } x>x_{0}\end{cases}
$$

where $n$ and $x_{0}$ are again given by Equation (14) and Equation (16), respectively. However, since $x_{0}>0$, Equation (16) requires $a>b$. In summary, for $a>b$, the solution $\hat{v}(x)$ deceases to zero at a point $x_{0}$, and is taken to be equal to zero beyond that point. Again see figure 12 .

The power law solution fails only for $a=b$ (including the case $a=b=1$, which was the original version of the continuum model [23]). In this third case the solution is exponential,

$$
\hat{v}(x)=\exp \left(-x(a k)^{\frac{-1}{1+a}}\right),
$$

which may be obtained from Equation (13) by taking the limit as $n$ tends to infinity (corresponding to $(a-b) \rightarrow 0$ ), or more directly by substitution.

We see that the indices $a$ and $b$ determine what kind of solution is found, that is; power law, truncated power law or exponential, as illustrated in Figure 12.

\subsection{Definitions and results for localization lengths}

What formula for the localization length emerges from these solutions? There are a number of definitions for the localization length which might be conventionally associated with a given velocity profile $\hat{v}(x)$. Firstly, the internal definition for the case of exponential localization, mentioned in section 2, may be generalized as

$$
\hat{v}\left(l_{e}\right)=\frac{1}{e}
$$

(This corresponds to $V / e$ in physical units.) In a similar manner it is possible to define $l_{1 / 10}$,

$$
\hat{v}\left(l_{1 / 10}\right)=\frac{1}{10}
$$

or localization lengths for other fractions.

Alternatively (and equivalently to Equation (19) in the case of an exponential velocity profile) we may use

$$
\left|\hat{v}(0) / \frac{d \hat{v}}{d x}\right|_{x=0} \mid=l .
$$


In the case $a>b$, in which $\hat{v}(x)$ vanishes beyond some point $x_{0}$, one could use the smallest $l$ for which

$$
\hat{v}(l)=0 .
$$

This might also be applied to the case of Couette geometry, which in certain cases can provide a profile that vanishes at some point, even for $a \leq b$ [24].

For present purposes the two definitions Equation (19) and Equation (21) are preferable (with a possible third candidate which we propose for the first time below), and the choice between them is rather arbitrary.

The definition provided by Equation (21) leads to the following formula for the localisation length, arising out of the solutions developed above, and happily common to all cases (i.e. all positive values of $a$ and $b$ ),

$$
l=\left(\frac{a(1+b)}{1+a} k\right)^{\frac{1}{1+a}}
$$

Recall that $k$ is given by Equation (10) and contains a velocity dependence, so the localization length scales with velocity as

$$
l \propto V^{\frac{a-b}{1+a}} .
$$

Note also the incorporation of $c_{v}$ and $c_{d}$ in $k$ : we see that the localization length increases with $c_{v}$ and decreases with $c_{d}$. When $c_{d}=0$ (no wall drag) there is no localization. It is this statement that places the model in stark contrast to the occurrence of localization in quasistatic simulations, in which wall drag is absent.

For the case of exponential localization $(a=b)$ Equation (23) reduces to

$$
l=\left(a \frac{c_{v}}{c_{d}}\right)^{\frac{1}{1+a}} .
$$

The velocity dependence of Equation (23) (contained in $k$, see Equation (10)) is absent, as was reported in the initial formulation of the continuum model [23].

When a localization length needs to be extracted from numerical or experimental data yet another definition may be useful, as the integration over a velocity profile reduces the effect of scatter in the data:

$$
l_{\text {int }}=\int_{0}^{L} \hat{v}(x) d x .
$$

Note that $l_{\text {int }}$ has indeed the dimension of a length since $\hat{v}(x)$ is dimensionless, having been scaled by the boundary velocity $V$. Inserting the three different solutions 
$\hat{v}(x)$ of the continuum model (Equations (13), (17) and (18)) results in one common expression for the localization length $l_{\text {int }}$. It is given, in terms of the previous definition of $l$ (Equations (21) and (23)), as

$$
l_{\text {int }} / l=(1+a) /(1+2 a-b) .
$$

A certain amount of irreducible algebraic clutter should not detract from the very elementary nature of this treatment. It was initially developed for the case $a=b=1$, leading to exponential localization, and seemed sufficient to offer an explanation of the results of Debrégeas et al. (section 2). However, the further experiments that we have mentioned indicate the need for, at least, the solution that corresponds to $a<b$, leading to a decrease of localization length with boundary velocity. This arose first in the work of the Leiden group, who investigated numerical solutions of Equation (9) with finite boundary conditions corresponding to their experiments (section 3.3).

Of course, all of the experiments use finite samples (finite $L$ ). The analytic solution that we have developed is for $L \rightarrow \infty$, and nothing as simple as what we have seen emerges for finite $L$ [26], except for the original case of $a=b=1$. For this, Equation (9) is linear and has, for example, the solution in the form of a sinh function [23], appropriate to the experimental condition of figure 6 (although it was not in the end found to be applicable to that experiment).

The main qualitative conclusions of the analysis of the continuum model - that wall drag induces localization in that model (and the internal viscous dissipation opposes it) and that there is a velocity-dependence determined by $a$ and $b$, must now be brought into closer contact with experiment.

\section{Brief comparison with experimental data}

Let us first of all examine the results which exposed a velocity dependence of localization. These were first obtained by the Leiden group, using the apparatus that we have described in section 3.3. Their model is essentially equivalent to that of the previous section, but using numerical methods and the appropriate boundary conditions for two moving boundaries [28, 30]. (Note however that, provided the two boundary velocities are equal and opposite, half of the system is equivalent to the case that we have chosen, by symmetry. That is, $v=0$ at the midpoint.)

The parameter $b$ was determined by the group as $b=0.67$ using an experimental technique developed by the Denkov group [19]. It consists of measuring the torque 
that is required for dragging a confined monolayer of bubbles over a smooth plate. Using this value for $b$ (for its significance see section 15), very good agreement with observed velocity profiles was obtained for the parameter value $a=0.36$.

The Leiden group also succeeded in obtaining an independent estimate of $a$ in reasonable agreement with these values from shearing their foam in a Couette rheometer and fitting their data to the Herschel-Bulkley model.

Using the above values for $a$ and $b$ in the continuum model expression for the variation of localization length with the velocity at the moving boundary (Equation (24) and Equation (27)) yields $l \propto V^{-0.23}$. This is the scaling relationship that was already shown to hold for the Leiden data (see section 3.3, Figure 7).

The further measurements of the group of Dennin added some further dimensions to our understanding. They showed that when a Bragg bubble raft is used, for which no wall drag is expected, localization is not observed [39]; this is an important element of support for the continuum model.

In these ways our understanding of shear localization in 2D foam has progressed considerably, but puzzles have remained.

- Why does the original Debrégeas experiment show no velocity dependence? Are we to conclude that $a=b$ in that foam sample? This remains a possibility - neither quantity was independently estimated - but it seems unlikely, in the light of other experiments.

- What is the significance of the additional feature observed under some conditions in Couette geometry, namely the discontinuity of the derivative of velocity profile with respect to radial position?

- How are we to resolve the apparent discrepancy between continuum modeling, and the repeated finding of localization in quasistatic simulations [31, 32, 33, 34, 35]? To clarify this issue once more, note that the $V \rightarrow 0$ limit of continuum theory takes the localization length either to zero or to infinity, unless $a=b$, which we no longer consider to be correct for real foams.

Before returning to some of these matters, we will deal briefly with the transient regime. 


\section{Transient and history-dependent effects}

In the original analysis of the continuum model [23], the full time-dependent form of its governing Equation (6) was used, although restricted to the case $a=b=1$. Rather than finding the steady state directly, the time-dependent solution was examined for the case in which the boundary velocity is instantaneously increased from zero to the finite value $V$ at $t=0$. Steady shear is suddenly switched on, as in most experiments. This results in quite a rich scenario, in which the transient solution goes through various forms before settling down in the steady state.

Whereas the eventual steady state is described in terms of a velocity profile $v(x)$, with the displacement profile $u(x)$ losing any significance, we need to think of both (and indeed local strain and stress also) when dealing with the transient behaviour. Velocity and displacement are, of course, connected by $v(x)=\frac{d}{d t} u(x)$.

Since there has been no experimental engagement with the results, we will describe them only briefly here, referring to the original reference [23] for various diagrams that present the detail.

The boundary velocity $V$ is switched on at $t=0$, at which point it is assumed that the system is described by $u=v=0$ everywhere, except at the boundary. Its response is described by the force balance equation which has the form

$$
\text { velocity }=F(\text { derivative of strain })+G \text { (derivative of strain-rate) }
$$

where $F$ and $G$ arise from the "elastic-plastic" and "viscous" terms of Equation (6), based on the Herschel-Bulkley (or in this case, Bingham, for $a=1$ ) relation. It is possible to interpret the behaviour of the system, as described by the numerical integration of Equation (6) in time, with simple mathematical arguments based on approximations, as follows.

Initially the $F$-term vanishes. The equation reverts to the form of Equation (9) which we have previously adduced to the steady state. Rather paradoxically, the solution immediately "jumps" to the same exponentially localized form, Equation (18).

Within the present theory this jump is instantaneous. This cannot be physically acceptable. It is presumed that the neglect of inertia is responsible. The propagation of a change of velocity profile on some short time-scale may be a feature of future experimental interest.

Switching on $v(x)$ in this manner entails thereafter the progressive development 
of displacement $u(x)$, increasing from zero, and consequent strain, giving rise to a finite value of the $F$-term in our equation. For low enough boundary velocity $V$, this grows to dominate, so that the profile of $v(x)$ and $u(x)$ evolve from an exponential profile towards a linear form, approximating the quasi-static equilibrium solution with constant stress and strain for linear elasticity.

This form in turn is undermined by the arrival of the strain value at which the stress approaches the yield (or limit) stress. Then the elastic-plastic $F$-term must again diminish (eventually to zero) and the solution collapses back to its steady-state form, exponential in the present case.

This appealing scenario, in which first $G$, then $F$, then $G$ again dominates, has not yet been tested experimentally, but it does seem to correspond qualitatively to what is seen, for example, in the video of the original Debrégeas observationsł.

More generally one may choose to implement some experimental protocol in which the boundary velocity $V$ is some function of time. In the primitive continuum model that we have described, the steady state is unique. It is independent of the way in which $V$ has been varied, provided that it asymptotes to the same value. However, we shall see in section 10 that this is no longer true when certain refinements of the model are introduced, and the time-dependent equation again becomes important.

One caveat must be continually borne in mind: the equations given up to this point do not admit a reversal of the sign of the local strain-rate anywhere. This is not just conventional: hysteretic effects need to be included in the more general case.

\section{Distinct yield and limit stresses}

In this section we introduce an additional factor which seems necessary to fully account for the experiments. It has been recognised for a long time that the (local) yield stress, at which continuous shearing commences, is slightly greater than the eventual limit stress. This was already sketched in Figure 8.

In previous sections this feature was not incorporated in the model. Instead the local stress was taken to be a monotonic function of strain and there was no distinction to be made between local yield and limit stresses.

Important qualitative consequences follow from having distinct yield and limit stresses. Figure 13 provides an immediate picture of what is suggested here - the $\ddagger$ http://tel.archives-ouvertes.fr/docs/00/04/56/98/HTML/Manip.mpg, we thank A. Kabla for this link 


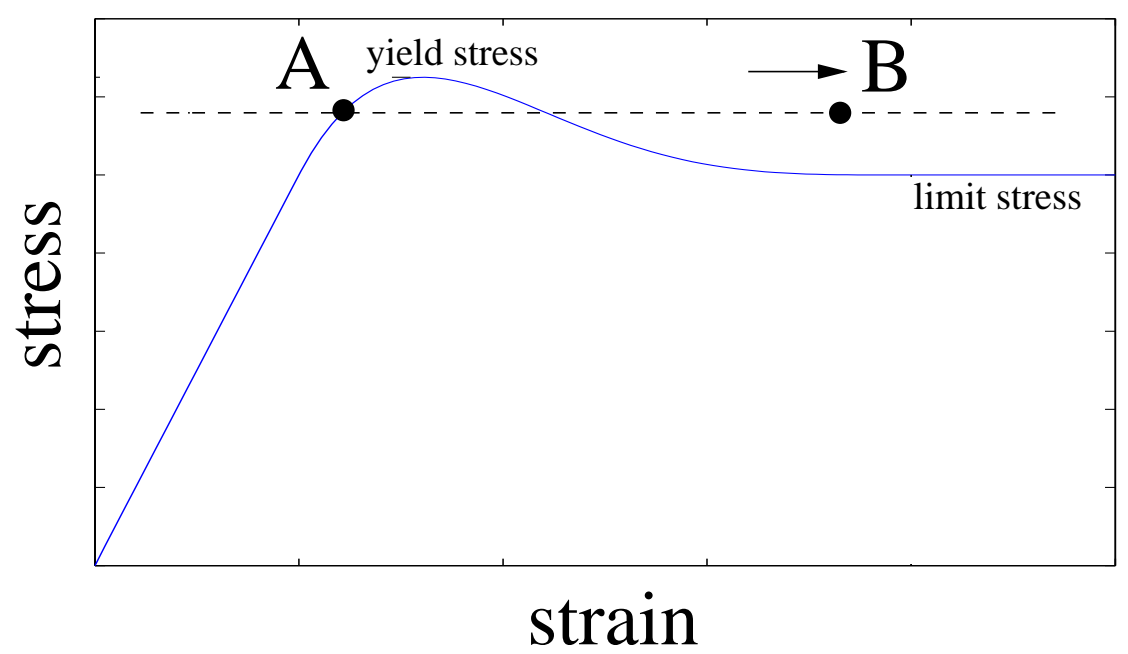

Figure 13. The existence of distinct yield and limit stress in the quasi-static stress-strain relationship allows, at low velocities, for the coexistence of a static (corresponding to $\mathrm{A}$ ) and a shearing region (corresponding to $\mathrm{B}$ ). Stress at the A/B boundary is the same, due to the additional dissipative term in the shearing region.

coexistence of static and shearing region with the same stress at the point of transition from one to the other. The effects, which are most marked below a critical boundary velocity defined below, are as follows.

- The steady state may include a part which is static, coexisting with a shearing region.

- The derivative $\frac{d v}{d x}$ is finite at the boundary between shearing and static regions (see figures 5 and 14).

- The steady state is no longer unique, but rather is dependent on history, that is, the previous variation of the boundary velocity $V$.

The coexistence illustrated by Figures 13 and 14 is not to be confused with that which was encountered earlier for the solution of the continuum model in the (so far unphysical) case $a>b$. In the generalisation of the continuum model that is considered here, it may occur for $a \leq b$ and indeed is not much dependent on the details of the model. 


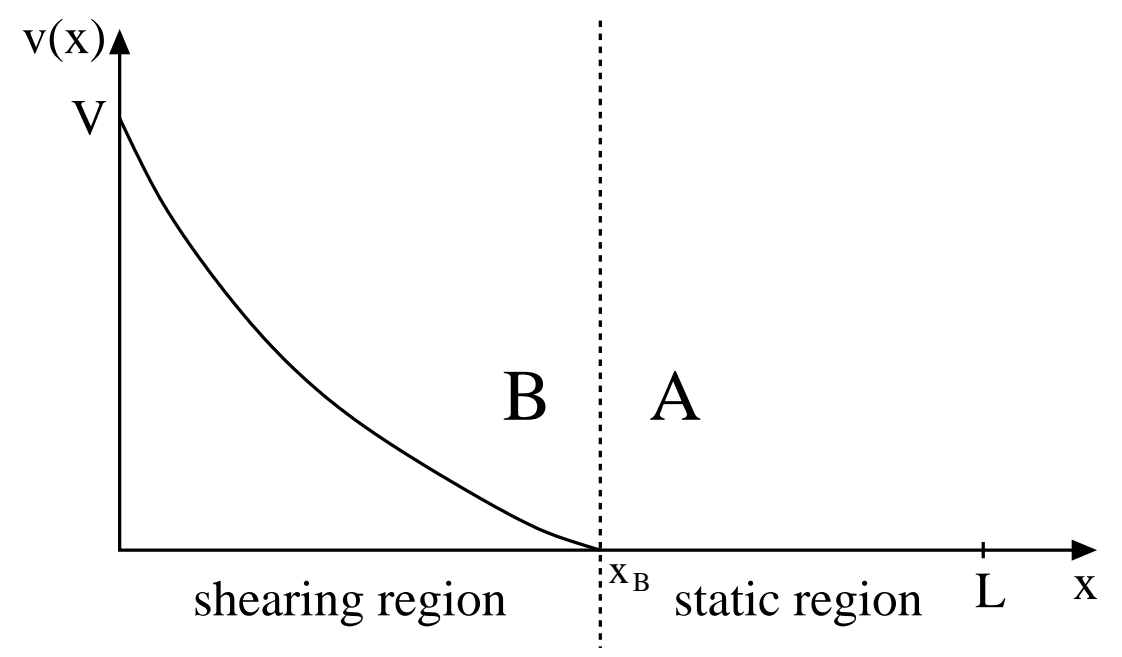

Figure 14. Coexistence of sheared and static regions is possible when yield and limit stress are distinct (cf. Figure 13).

Nor is it to be confused with a similar effect for Couette geometry [24], which is induced by the circular geometry. The first clue to the necessity to refine the model was nevertheless provided by the experiments of the group of Dennin [41, 42] which had circular Couette geometry. An additional feature emerged, not predicted by that model - the finite first derivative $\frac{d v}{d x}$ at the edge of the shearing region to which we have just referred. Revisiting the original derivation of the continuum model, one may directly rule out this effect within that model, by considering the balance of forces at the point in question, separating moving and static regions. In due course, it was shown by the Dublin group [60] that replacing the elastic/plastic shape function $f(u)$ by one which had distinct yield and limit stresses, and proceeding as before, allowed the required feature. This interpretation was as given in figure 13. There must be equal stresses on the two sides of the boundary point between shearing and static regions, and these values correspond to the two points $\mathrm{A}$ and $\mathrm{B}$ on the diagram.

In further pursuing this phenomenon, we shall revert to the straightforward case of simple shear geometry, and ask: what solutions of this kind, as in Figure 14, can exist for given $V$ ?

The stress matching illustrated by figure 13 can be accomplished for any stress value lying between the limit and the yield stress, by introducing the appropriate value of $\frac{d v}{d x}$ at the boundary point $x_{B}$. The solution is therefore not unique. In further 
discussing this, we take the sample width $L$ to infinity, as before.

Recall that we have at our disposal the exact solution for $v(x)$ in the limit stress regime, from Equation (9). This has a single disposable parameter which was previously fixed by the condition $v \rightarrow 0$ as $x \rightarrow \infty$ (the condition $v(0)=1$ still holds). But now we can instead allow $v$ to go to zero at a point $x_{B}$, as in Figure 14, proceed to examine the value of $\frac{d v}{d x}$ at that point, and apply the condition that stress is a continuous function of $x$. The solution may be stitched together at this point, provided that the viscous stress term is not too large, that is, provided that

$$
c_{v}\left|\dot{\gamma}\left(x_{B}\right)\right|^{a} \leq \text { (yield stress - limit stress), }
$$

at the point in question, where $v$ is zero. For convenience, let us refer to the right hand side of Equation (29) as $\Delta$. There is a continuous range of possible solutions defined by the above equation. For each of these we may define a localization length $l$, in terms of the derivative of $v$ at $x=0$ (Equation (21)). The range of allowed solutions is illustrated by Figure 15 in terms of $l$, for the primitive $(a=b=1)$ continuum model, and another example $(a=0.5, b=1)$.

In each case the upper curve $l^{+}(v)$ corresponds to $x_{B} \rightarrow \infty$, and it is identical to the solution found in the earlier version of the model, with yield stress being identical to limit stress.

The lower curve $l^{-}(v)$, in which shear is most localized, has shearing and static regions (as have the solutions between the two bounds). This corresponds to equality in Equation (29). Note that it needs to be calculated numerically [50].

The range of possible solutions is large for low $V$, that is, for

$$
V<\Delta / \sqrt{c_{d} c_{v}},
$$

as may be estimated by making a linear approximation for the lower values of $l$ close to $V=0$, and finding the intersection of this with the upper curve. This value is for the primitive model $(a=b=1)$. For the general model it is given by [50]

$$
V<\Delta^{\frac{a+1}{a(1+b)}}\left(\frac{a(1+b)}{1+a}\right)^{\frac{1}{1+b}}\left(\frac{1}{c_{d}\left(c_{v}\right)^{\frac{1}{a}}}\right)^{\frac{1}{1+b}} .
$$

Having established this range of possibilities, we return to the question: which solution is selected in an experiment?

This question has not yet been fully answered, but calculations with the time-dependent model (for Couette geometry) have suggested that the eventual 

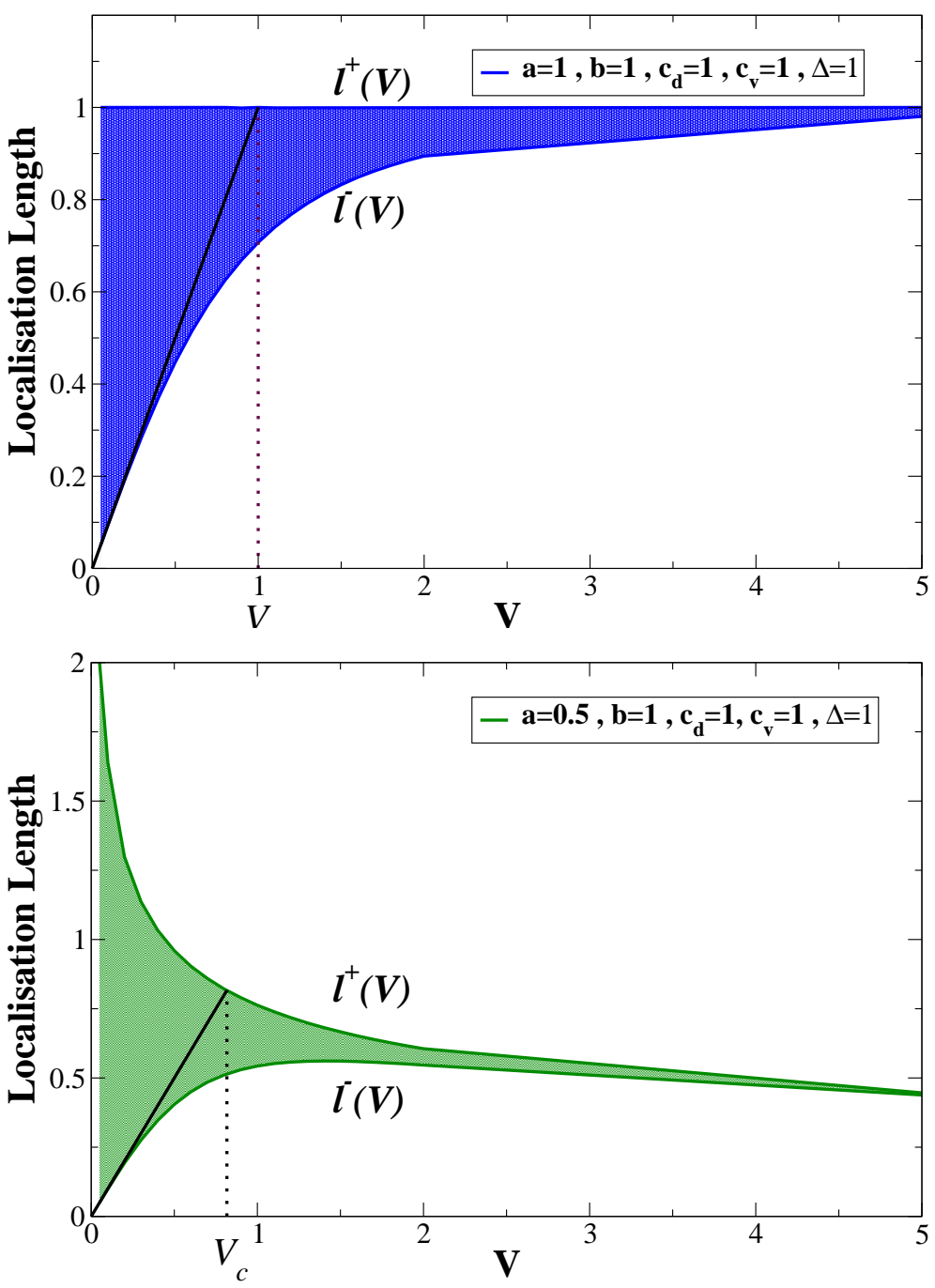

Figure 15. The shaded regions correspond to allowed values of localization length $l$ as a function of boundary velocity $V$, as given by continuum theory with distinct values for yield and limit stress. For this illustration exponents $a$ and $b$ are set to $a=1, b=1$ (top) and $a=0.5, b=1$ (bottom). In both cases the difference between yield and limit stress $\Delta$ was set to $\Delta=1$. $V_{c}$, as given by Equation (30) and Equation (31), respectively, serves as a rough estimate of the value of boundary velocity below which there is a wide range of possible solutions. 
steady state is history-dependent [60]. The implications for future simulations and experiments are clear: the protocol of $V(t)$ should be varied, in search for these solutions.

Before pursuing the course of detailed simulations which incorporate dissipation due to wall drag, in the next section we demonstrate that a localized solution of the kind shown above can be constructed, even if the wall drag is absent.

\section{A mechanism for localization in the absence of wall drag}

The present analysis has further implications for addressing the key question of: why are finite values for the localization length found in quasistatic simulations [31, 32, 33, 34, 35] when the standard continuum theory predicts otherwise?

Here we will show that the addition of the effects of the stress overshoot (see Figure 8) to the standard theory resolve this apparent discrepancy, in the limit of $V \rightarrow 0$.

As done in Equation (6), on dimensional grounds we may relate the viscous drag force (per unit area) at point $x$ to the local stress (a force per length in 2D) at this point:

$$
-c_{d} v(x)^{b}=\frac{d \sigma(x)}{d x}
$$

Clearly, as the boundary velocity $V \rightarrow 0$, so too does the local velocity at each point $x$. The left hand side of eq. 32 therefore tends to zero, leading us to the conclusion that the stress must be constant across the sample in the quasistatic regime. In a real foam, where there is a distribution of bubble sizes inducing inhomogeneity, this argument may not be accurate; but in a continuum, there are no discrete elements (bubbles), so the sample is homogenous in space.

From eq $\mathrm{X}$ we see that a constant stress also implies that the local strain rate is constant across the sample. This entails a linear velocity profile of the following form:

$$
v(x)=V(1-x / l),
$$

where $l$ is the localisation length as given by eq. 21. An example of such a velocity profile is show in Fig. 16.

Like in the previous section, we may ask ourselves: for a given $V$, what range of localisation lengths is possible for solutions of the form of eq. 33? Clearly, the 


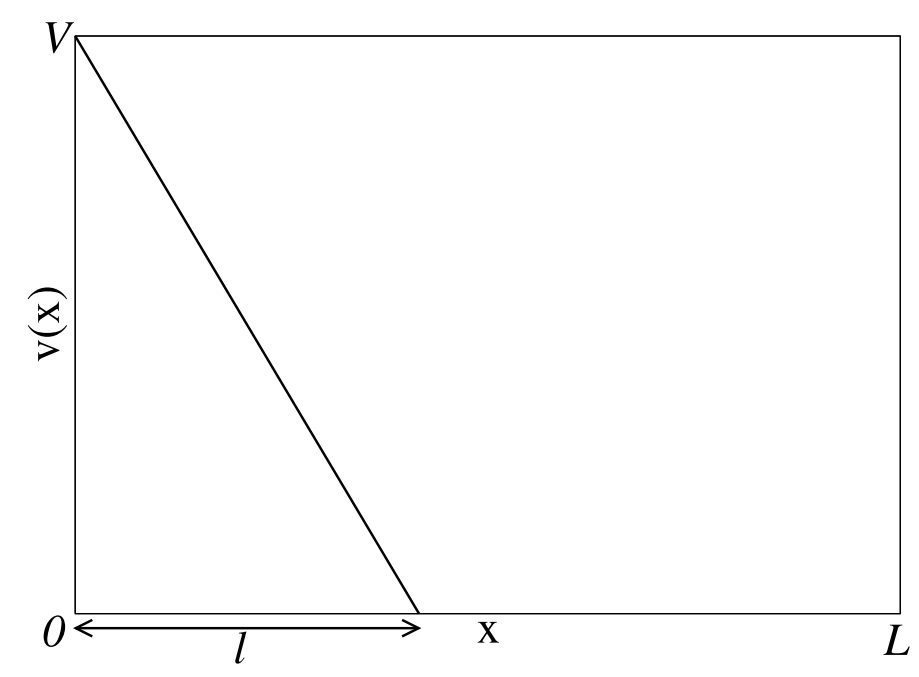

Figure 16. A linear velocity profile, which occurs when the stress becomes homogeneous across the sample. $l$ is the localisation length, which may take on a range of values (see Figure 17)

maximum possible localisation length $l$ is the system size $L$. As $L \rightarrow \infty$, this corresponds to $c_{v}|\dot{\gamma}|^{a}=0$ (see Equation (29)).

The corresponding lower bound to the range of possible localisation lengths is given by $c_{v}|\dot{\gamma}|^{a}=\Delta$ (see Equation (29)). From Figure ??, one can see that $|\dot{\epsilon}|=V / l$. Combining these two expressions leads to the following form for the localisation length $l$ as a function of $V$ :

$$
l=\left(\frac{c_{v}}{\Delta}\right)^{1 / a} V
$$

This gives a straight line through the origin, which intersects the (constant) upper bound at a critical velocity $V_{c}$; see Figure 17 . The shaded region in this figure indicates the range of possible localisation lengths which are possible for low $V$. It suggests that in the quasistatic regime, the sample may adopt any localisation length between 0 and the system size $L$. Above the critical velocity $V_{c}$, the approximation that the velocity profile is linear is no longer accurate however, and so the standard continuum theory applies once again.

These observations are certainly consistent with the quasistatic simulation results $[31,32,33,34,35]$ performed to date. We contest that the effect of distinct 


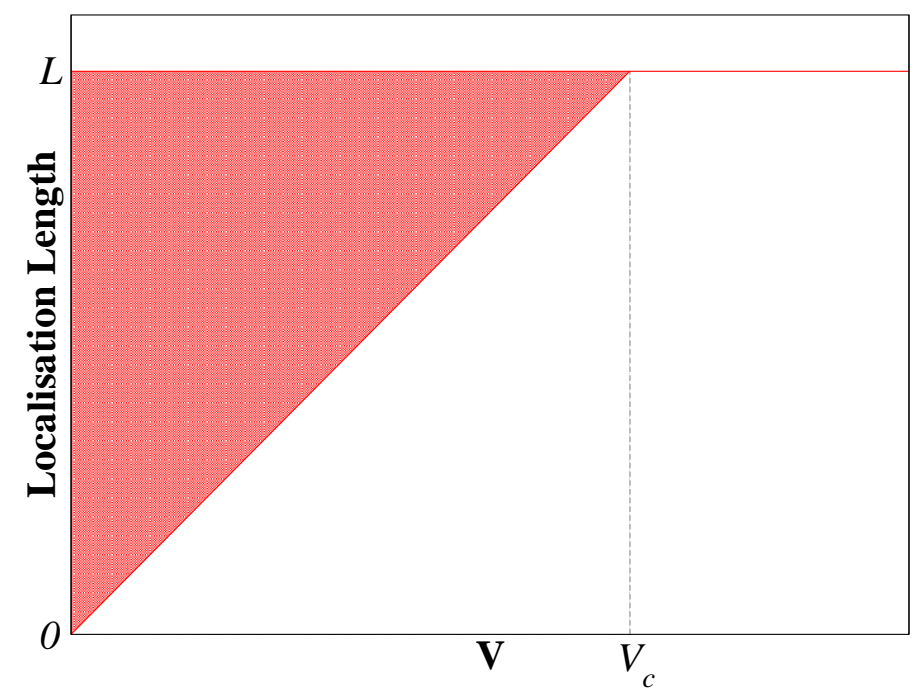

Figure 17. Localisation length as a function of boundary velocity $V$, in the limit of $V \rightarrow 0$. The shaded region indicates the allowable range of localisation lengths, as given by Equation (29).

yield and limit stresses is one possible mechanism for shear localisation in the absence of wall drag. Similar assertions have been made before: an argument which seems to point in the same direction is to be found in the original paper of Kabla and Debrégeas [31].

Of particular importance here is that these arguments can be extended to 3D (where there is also no wall drag present), thereby offering a mechanism for shear localisation in this case. A detailed discussion of this phenomenon however lies outside the scope of this review article, which is concerned with shear localisation in $2 \mathrm{D}$.

We therefore return to the question: what determines the allowable range of localisation lengths in a 2D foam? This will be pursued using detailed simulations that include dissipation, such as the viscous froth model [49] and the soft disk model [27]. To review all that has been done and is ongoing with such simulations would take us too far afield. Instead let us give some pertinent examples of recent results, in the next two sections, providing some interesting comparisons with the continuum model. 


\section{Some relevant results of the $2 \mathrm{D}$ viscous froth model}

\subsection{The model}

The $2 \mathrm{D}$ viscous froth model was formulated quite some time ago by one of the present authors as a "toy model" of primarily theoretical interest [61, 62]. It formed a bridge between two elementary physical models that had become standard: the quasistatic equilibrium model of a dry 2D foam, and the model of curvature-driven growth of a 2D cellular structure. Indeed it contains each of these, in different limits [49].

As is so often the case, this idealised conception, initially supposed to be physically unrealistic, turned out to have a significant physical realisation. It is now a useful starting point to describe the dynamics of $2 \mathrm{D}$ foams and related microfluidic systems $[49,63]$.

The model turns the traditional static cellular model of a $2 \mathrm{D}$ foam, in terms of a surface tension $\sigma$ and cell pressures $p$, into a dynamic one, by adding a viscous drag on a soap film (cell boundary), proportional to its local normal velocity. This is the "wall drag" to which we have repeatedly referred in this article, but here it relates to the detailed local motion of the soap films, rather than the average local speed of these films, as in the continuum model.

The equation of motion of a point $s$ on a soap film is given by

$$
\lambda v^{\perp}(s)=\Delta p-\sigma K(s)
$$

where $\lambda$ is the drag coefficient, $v^{\perp}(s)$ is the velocity in the direction normal to the boundary at $s, \Delta p$ is the pressure difference between two neighbouring bubbles, $\sigma$ is surface tension and $K(s)$ is the local curvature, see also Figure 18.

\subsection{Relationship to continuum model}

The viscous froth model is a natural candidate for a dynamic simulation of $2 \mathrm{D}$ foam rheometry, and this has been undertaken by Barry et al. [50]. We will summarise results in Section 12.3.

We shall see that localisation is found using this model and the results bear some relation to those of the continuum model, so that it is reasonable to ask: can the two models be related? At first, one is tempted to conclude that, if the viscous froth model is to be transposed into an equivalent continuum model, the latter can contain 


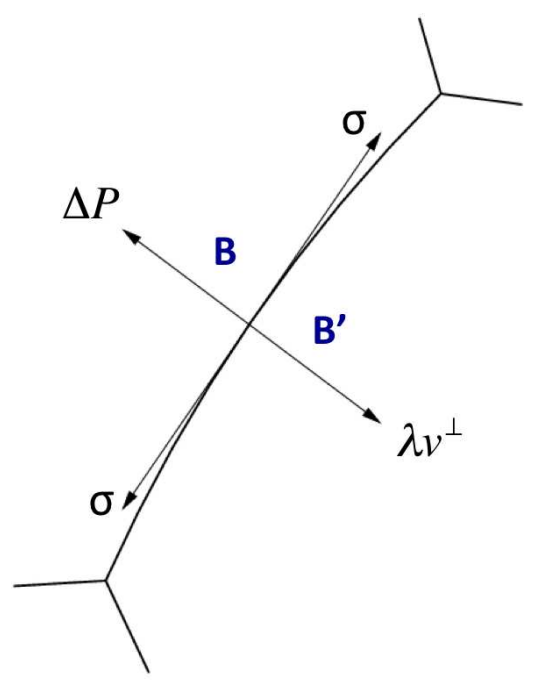

Figure 18. Sketch of the forces acting on a soap film in the viscous froth model. In a computer simulation such a curved film is approximated by straight line segments and Equation (35) is applied to the end points of the segments, with the exception of the vertices.

only the wall drag term, and not the term which represents internal dissipation in the Herschel-Bulkley relation. The results will suggest otherwise.

We first address the wall drag term. A local linear drag law in the viscous froth model will give $b=1$ in the continuum model, according to any simple averaging procedure. The drag constant $c_{d}$ of the continuum model expressed in terms of $\lambda$ of the viscous froth model may be estimated as

$$
c_{d}=\frac{1}{2} \sqrt{2 \sqrt{3} \bar{A}^{-1 / 2}} \lambda,
$$

where $\bar{A}$ is the mean cell area. This expression takes into account the number of cell walls per area, their average orientation, and the fact that in the continuum model the viscous drag is due only to the movement of the cell walls in the direction of applied shear [50].

The case of the index $a$ is altogether more problematical, as already noted. The results will suggest that $a$ is approximately 0.3 in the equivalent continuum model ([50] and Figure 21). We believe the interpretation of this is to be found in the concept of "relaxation stress (or strain)", which has been introduced in the context 
of foams by the Leiden group [29].

The coefficient of the internal viscous contribution to stress $c_{v}$ can be written on dimensional grounds as the following product of the parameters of the viscous froth model,

$$
c_{v}=\hat{c_{v}} \sigma^{1-a} \lambda^{a} \bar{A}^{a-1 / 2} .
$$

Here $\hat{c_{v}}$ is a dimensionless quantity of order unity, possibly dependent on the cell size distribution.

In the simulations, as in our previous analysis, shear is imposed on the foam by moving one boundary at velocity $V$, while the opposing boundary (at distance $L)$ is kept fixed. Soap films in contact with any of the boundaries remain in contact throughout the simulation, corresponding to no-slip boundary conditions. The overall (nominal) shear rate $\dot{\gamma}$ is thus given by $\dot{\gamma}=V / L$.

Together with Equation (37) this leads to the following formulation for the Herschel-Bulkley relation in terms of the parameters of the viscous froth model,

$$
S=S_{Y}+\hat{c_{v}} \sigma^{1-a} \bar{A}^{a-1 / 2} L^{-a}(\lambda V)^{a} .
$$

It is thus the product of drag coefficient $\lambda$ and boundary velocity $V$ that will play an important role when interpreting the results of the viscous froth shear simulations with the continuum model.

The immediate interest of the results may lie not so much where they conform to expectations based on elementary continuum theory but rather where they do not. For low values of the product $\lambda V$ there are discrepancies, which we believe are attributable to two causes.

We have already signaled one of these effects, that due to the difference of yield and limit stress (section 10). Secondly, as the effect of the drag term becomes less, the effect of disorder may become more significant, in that the yield stress may be considered to have a spatial variation.

\subsection{Results of computer simulations}

Simulations were performed on five different foam samples of similar cell area distribution, each consisting of 100 cells, and generated using the Surface Evolver software $[64,65]$. Shear is applied by moving the top boundary incrementally at each time step and then computing the resulting movement of all cell boundaries by use 


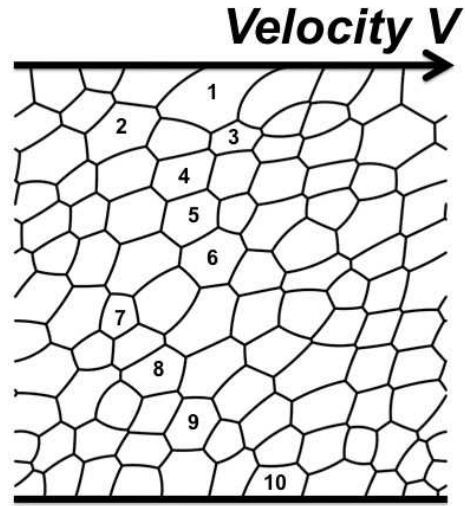

Strain $=2.0$

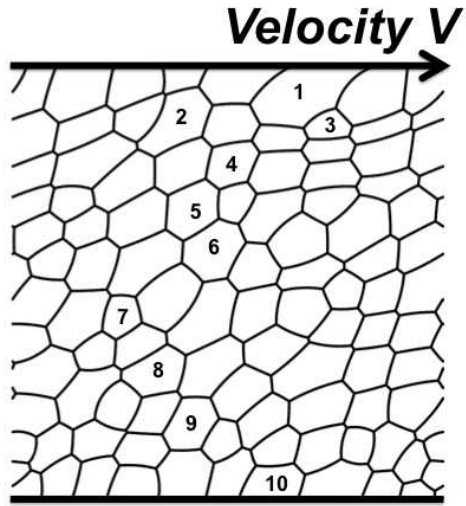

Strain $=2.2$

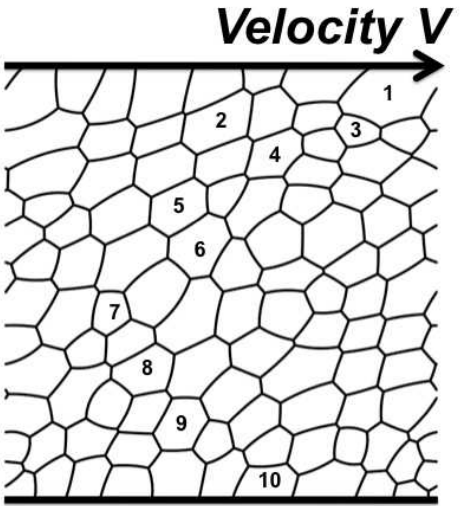

Strain $=2.4$

Figure 19. Snapshots of a shear simulation using the viscous froth model. The cell numbers help in identifying topological changes. The foam is periodic in the direction of applied shear.

of Equation (35). A velocity profile of the flowing foam is obtained by averaging the horizontal displacement of cell centres. The simulation is taken to have reached a steady state when the velocity profiles no longer appreciably change as shear is increased (this was found to require less than an applied strain of one). Steady-state velocity profiles were then averaged for values of strain between 1 and 10 and it is these steady-state profiles that were analysed for comparison with continuum theory.

The simulations exhibit clear evidence of localization, as indicated by Figure 19, where the displacement of bubbles 1 to 4 in the direction of imposed shear is contrasted with that of bubbles 5 to 10 which, on average, do not move. The region of localization of flow is almost always found to occur next to the moving, as opposed to the static, boundary.

Typical velocity profiles from simulations are shown in Figure 20, for different values of $\lambda V$, the product of drag coefficient $\lambda$ (see Equation (35)) and boundary velocity. Localization increases with $\lambda V$.

The analytic form of the velocity profiles of Figure 20 is hard to discern from the existing simulations, due to the small system size. Future computations involving

$\S$ This does not hold for simulations of monodisperse foams where the localization length is one bubble diameter, independent of $\lambda V$. Note that this is similar to experimental findings for sheared monodisperse foams, see section 3 . 


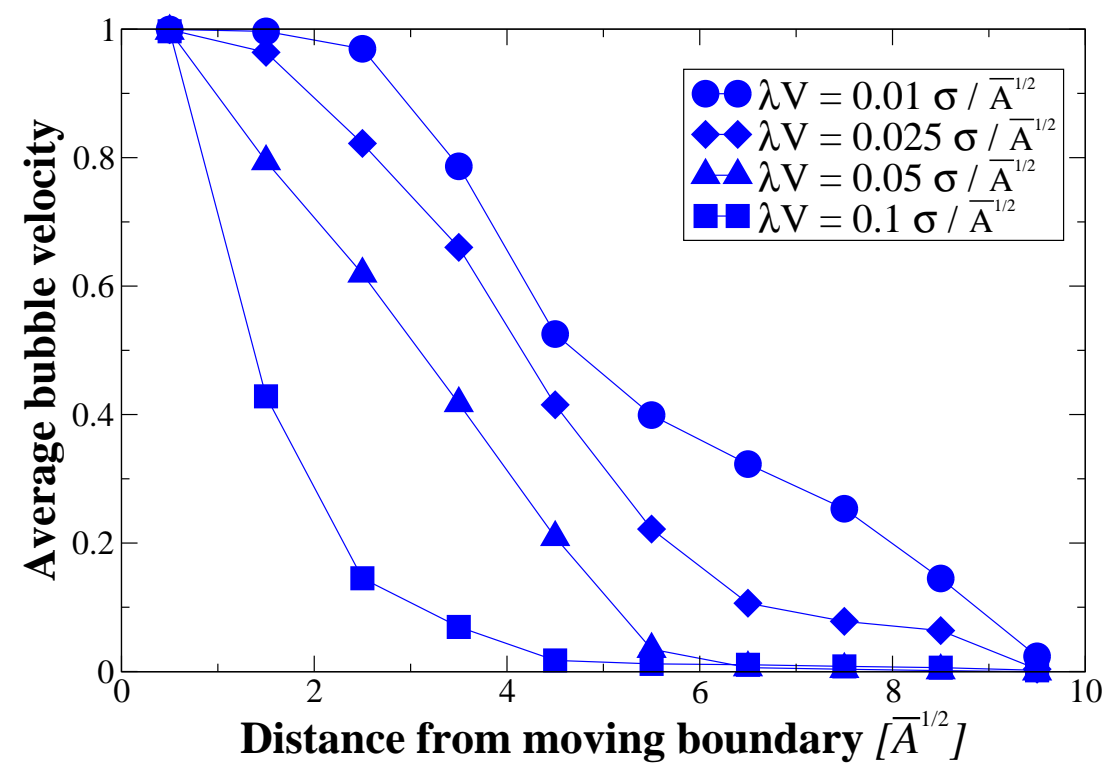

Figure 20. Velocity profiles obtained from individual viscous froth simulations using a sample of 100 cells. Increasing the product of drag coefficient $\lambda$ (see Equation (35)) and velocity $V$ of the moving boundary leads to localization near the moving boundary of the foam sample. Localization lengths can be extracted by integrating the profiles over the entire sample width, see Equation (26) and Equation (27).

larger numbers of bubbles may be more illuminating. However, it is noted that the profiles have an approximately linear form before a subsequent tail-off.

The scatter in the data makes the integral definition (Equation (26) and Equation (27)), the best choice for computing a localization length $l$. Figure 21 shows the variation of $l$ with $\lambda V$. For small values of $\lambda V$ there is a wide variation of the localization length $l$, as computed for five different realisations of foams containing 100 cells each and having a similar distribution of cell areas. As $\lambda V$ increases the range of $l$ values narrows.

How can we account for this rich behaviour? We contend that the idea of distinct yield and limit stresses, as discussed in Section 10, can provide answers. Figure 21 is qualitatively similar to Figure 15(b) and supports the assertion that, for low velocities, the localization length can take on any value between zero and infinity. 


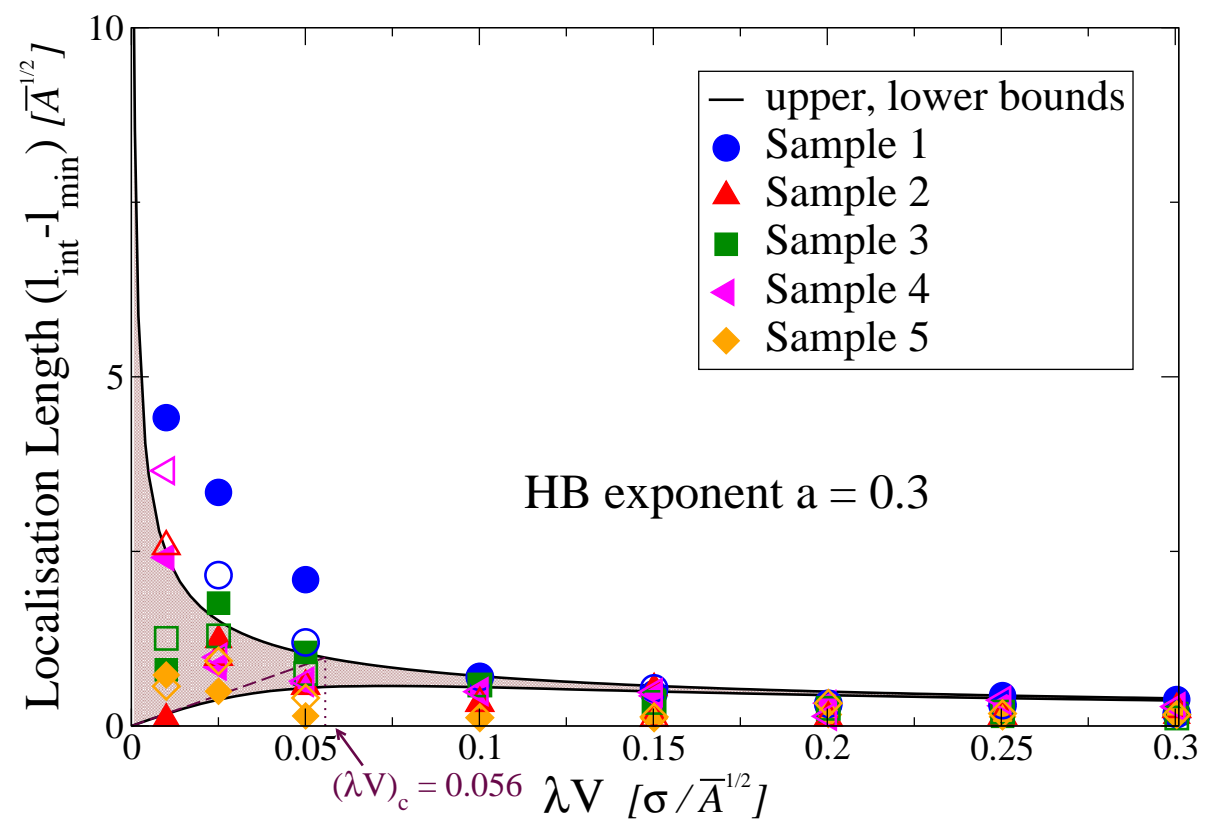

Figure 21. Localization length for a range of values for the product $\lambda V$ of wall drag coefficient $\lambda$ and velocity $V$ of the moving boundary. The data was computed for five different foam samples, each containing 100 cells and having a similar second moment $\mu_{2}(A)$ of the cell area distribution $\left(\mu_{2}(A)=0.13 \pm 0.002\right)$. The shaded region marks the allowed region for the localization length as given by the continuum theory for the case of distinct values for yield and limit stresses. It has been computed by setting the Herschel-Bulkely exponent $a=0.3$ and the difference between yield and limit stress to $\Delta=0.11 \sigma \bar{A}^{-1 / 2} .(\lambda V)_{c}=0.056 \sigma \bar{A}^{-1 / 2}$ is an estimate below which value the localization length becomes essentially independent of $(\lambda V)$.

The upper bound for localization lengths $l^{+}(V)$ (see Equation $(23)$ ) can be expressed as a function of $\lambda V$ by using the relevant expressions of $c_{d}$ and $c_{v}$ for the viscous froth model (Equation (36) and Equation (37)) [50]. This results in

$$
l^{+}(\lambda V)=\left(\frac{2 a \hat{c_{v}} \sigma^{1-a} \bar{A}^{a-1 / 2}}{(1+a) \hat{c_{d}}}\right)^{\frac{1}{1+a}}(\lambda V)^{\frac{a-1}{1+a}} .
$$

Similarly, an expression for the rough estimate for the critical value of $\lambda V$ can be found, below which localization can essentially occur anywhere in the sample (based 
on Equation (31))[50]. It is given by

$$
(\lambda V)_{c}=\sqrt{\frac{2 a \Delta^{\frac{1+a}{a}}\left(\hat{c_{v}} \sigma^{1-a} \bar{A}^{a-1 / 2}\right)^{-\frac{1}{a}}}{(1+a) \hat{c_{d}}}} .
$$

The above equations for $l^{+}(\lambda V)$ and $(\lambda V)_{c}$ contain three unknown parameters, namely $\hat{c_{v}}, \Delta$ and $a$. The difference $\Delta$ of yield and limit stress refers to the stressstrain curve in the quasistatic limit. It was estimated from quasistatic simulations of foams using the Surface Evolver as $\Delta=0.11 \sigma \bar{A}^{-1 / 2}$ [50]. Setting the HerschelBulkely index to $a=0.3$ and the dimensionless constant, which should be of order unity, to $\hat{c_{v}}=0.26$ produces both upper and lower bounds $l^{+}$and $l^{-}$that look consistent with the results from the viscous froth simulations, as shown in Figure 21 [50].

As we have already said, it is not obvious that the viscous froth model should have properties consistent with the continuum model. At this stage we can only conclude that it does, and the detailed explanation remains to be found.

\section{Some relevant results of the soft disk model}

Durian used a simpler, non-cellular, representation of a 2D foam, in which the bubbles are represented by overlapping disks $[7,8]$, as shown in figure 22. This "toy" model (equally applicable to certain granular materials) is easy to program and lends itself readily to incorporation of ad hoc elastic and dissipative forces, and hence to rheology. We will call it the soft disk model for present purposes.

The model has been revisited by Langlois et al. [27], and others, with startling conclusions. It appears that the approximations made by Durian and others in the very early attempts at such a simulation were such as to render some of the results erroneous.

The simulation of Langlois et al. [27] uses elastic and dissipative forces that depend only linearly on displacements and velocities. The reasonable expectation would then be that $a=1$ should be found (as Durian did), but the new calculations give values of a much less than unity. And the reason cannot lie in the forces themselves. 


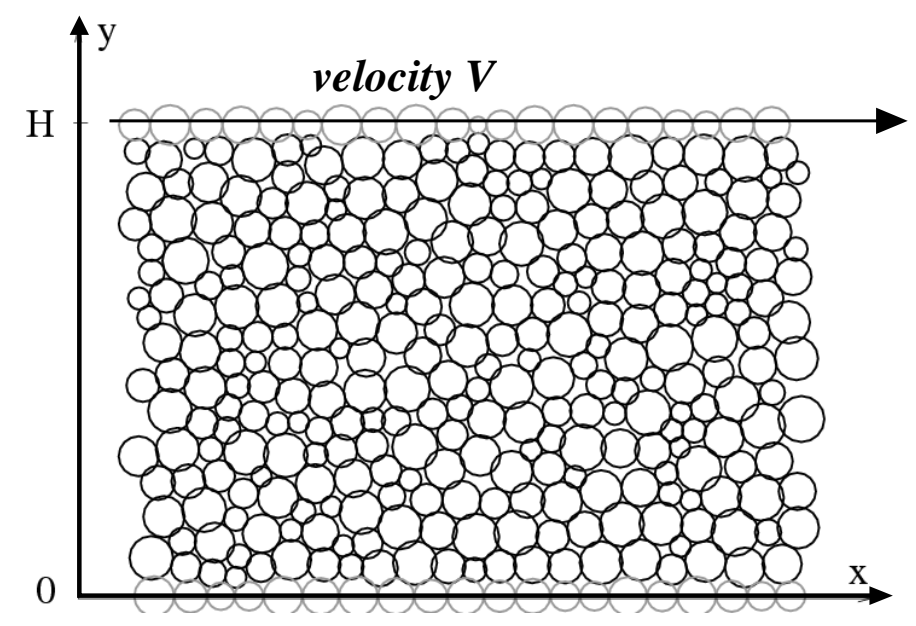

Figure 22. Snapshot of a shear simulation using the disk model. In the example shown the bubbles are confined between a static boundary at $y=0$ and a moving boundary at $y=H[27]$. The simulation uses periodic boundary conditions in the $\mathrm{x}$-direction only.

\subsection{Definition of the model}

The forces in the soft disk model are as follows. When two disks (representing two bubbles) overlap (and only then) they interact via a simple spring force, the displacement of the spring being the radial overlap (see Fig. 23).

The elastic repulsive force $\mathbf{F}_{\mathbf{n}}$ acting on bubble $i$ (centred at $\mathbf{r}_{\mathbf{i}}$, with radius $R_{i}$ ) due to bubble $j$ (centered at $\mathbf{r}_{\mathbf{j}}$, with radius $R_{j}$ ) is then given by

$$
\mathbf{F}_{\mathbf{n}}=\kappa \frac{2 R_{0}}{R_{i}+R_{j}} \Delta_{i j} \mathbf{n}_{\mathbf{i j}}
$$

Here $\kappa$ is the coefficient of elasticity, $\mathbf{n}_{\mathbf{i j}}$ is the normal vector between bubbles $i$ and $j$,

$$
\mathbf{n}_{\mathbf{i j}}=\frac{\mathbf{r}_{\mathbf{i}}-\mathbf{r}_{\mathbf{j}}}{\left|\mathbf{r}_{\mathbf{i}}-\mathbf{r}_{\mathbf{j}}\right|},
$$

and the overlap $\Delta_{i j}$ is given by

$$
\Delta_{i j}=\left\{\begin{aligned}
\left(R_{i}+R_{j}\right)-\left|\mathbf{r}_{\mathbf{i}}-\mathbf{r}_{\mathbf{j}}\right| & \text { if }\left(R_{i}+R_{j}\right)<\left|\mathbf{r}_{\mathbf{i}}-\mathbf{r}_{\mathbf{j}}\right| \\
0 & \text { otherwise }
\end{aligned}\right.
$$




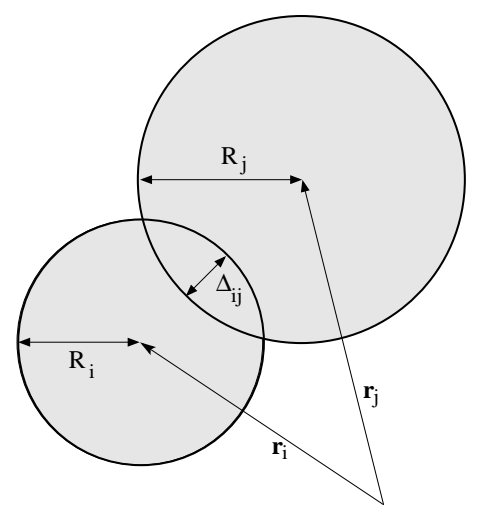

Figure 23. Overlap $\Delta_{i j}$ between two contacting bubbles of radii $R_{i}$ and $R_{j}$, located at $\mathbf{r}_{\mathbf{i}}$ and $\mathbf{r}_{\mathbf{j}}$, respectively.

(see Fig.23). The ratio $\frac{2 R_{0}}{R_{i}+R_{j}}$ in Equation (41), where $R_{0}$ is the average bubble radius of the entire bubble packing, takes into account that larger bubbles are easier to deform than smaller ones.

A real flowing foam dissipates energy by viscous friction in the films and Plateau borders separating the bubbles. The simplest expression, as used by Durian [8] and [27], represents the viscous force $\mathbf{F}_{\mathbf{b}}$ on bubble $i$ associated with a neighbouring bubble $j$ as

$$
\mathbf{F}_{\mathbf{b}}=-c_{b}\left(\mathbf{v}_{\mathbf{i}}-\mathbf{v}_{\mathbf{j}}\right)
$$

where $c_{b}$ is the dissipation constant for the bubble-bubble interaction and $\mathbf{v}_{\mathbf{i}}$ and $\mathbf{v}_{\mathbf{j}}$ are the respective bubble velocities.

Wall drag in the case of a foam confined between two plates adds an additional force on all moving bubbles. It is given by

$$
\mathbf{F}_{\mathbf{w d}}(\mathbf{r})=-c_{w d}|\mathbf{v}(\mathbf{r})|^{b} \frac{\mathbf{v}(\mathbf{r})}{|\mathbf{v}(\mathbf{r})|}
$$

where $c_{w d}$ is the wall drag constant. The exponent $b=1$ in the simulations of Langlois et al. [27].

\subsection{Deborah number}

The Deborah number is a dimensionless quantity that is often used in rheology. It is the ratio of a characteristic time associated with shear and some time characteristic 

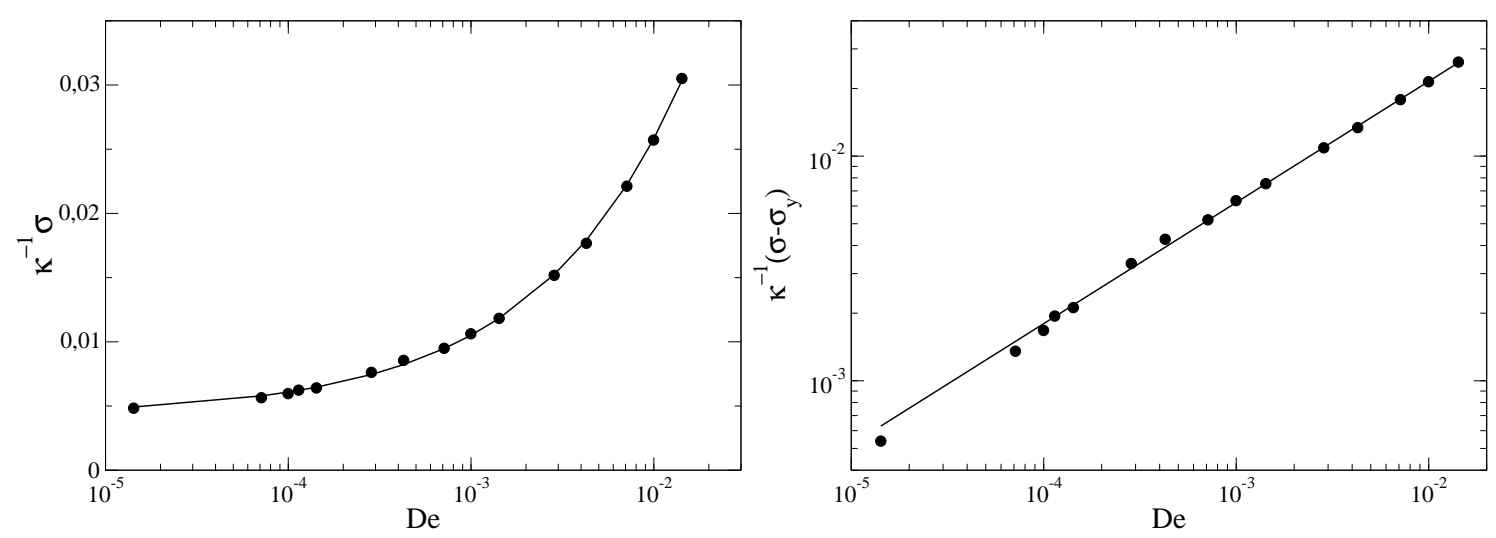

Figure 24. (a) Stress on the moving boundary as a function of the Deborah number De $=\dot{\gamma} c_{b} / \kappa$ (Equation (46)). The solid line is a fit to Equation (4) (re-expressed in terms of De), resulting in the Herschel-Bulkley exponent $a=$ $0.54 \pm 0.01$. In (b) we have subtracted the fitted value of the yield stress $S_{Y}$ from the data to show the power-law behaviour in a double logarithmic plot.

of the relaxation of the material [66]. Its explicit definition depends somewhat on convention, since the "relaxation time" could be interpreted or estimated in various ways, and the appropriate definition may vary according to the regime under consideration. One should be cautious, therefore, in asserting any immediate significance to a particular realisation of the Deborah number.

In the case of the bubble model without wall drag the Deborah number $D e$ was defined as [7, 27]

$$
D e=\dot{\gamma} \frac{c_{b}}{\kappa} .
$$

Whenever wall drag is present which depends linearly on bubble velocity $(b=1$ in Equation (45)), the following extension,

$$
D e^{\prime}=\dot{\gamma} \frac{c_{b}+c_{w d}}{\kappa},
$$

appears more appropriate since it takes into account the wall drag contribution to the local relaxation time which can be taken as the timescale of individual bubble rearrangements. 


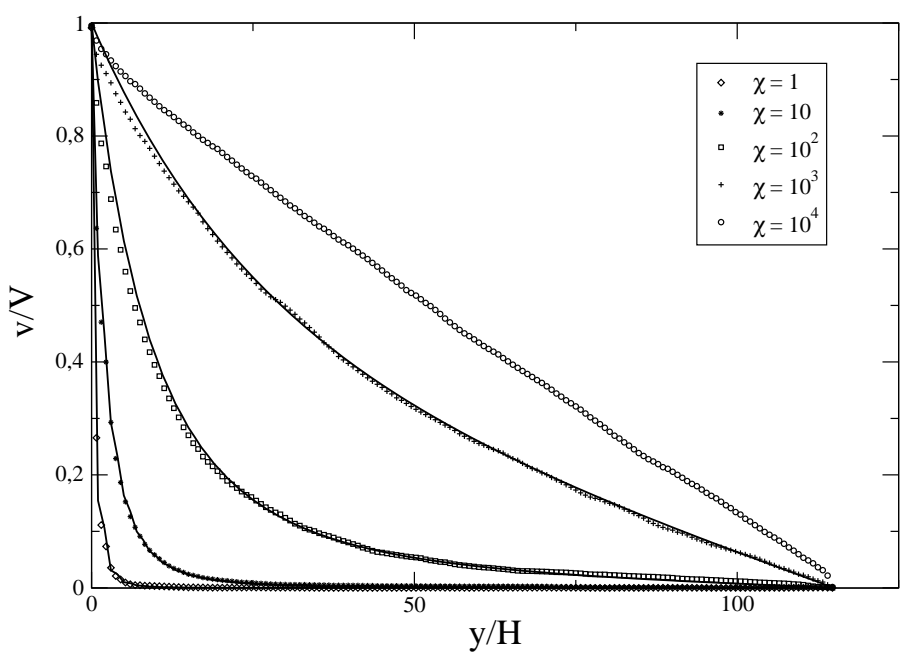

Figure 25. Normalized velocity profiles in the foam for various values of the ratio $\chi=c_{b} / c_{w d}$ of dissipation and wall drag constant (defined in Equation (44) and Equation (45), respectively). The localization of the shear on the moving wall increases as wall drag is increased, i.e. with decreasing $\chi$. The solid lines, which agree closely with the data, were obtained from numerical solutions of the continuum model, Equation (9), for values of $a=0.54$ for the Herschel-Bulkley exponent and $b=1$ for the exponent of the drag force [27].

\subsection{Results}

Computer simulations of the soft-disk model, as defined in section 13.1, were performed by Langlois et al. [27]. These simulations of assemblies of up to 10000 disks show that in the absence of wall drag the bubble model exhibits a Herschel-Bulkley relationship under applied shear with an exponent $a=0.54 \pm 0.01$ (Figure 24). Adding wall drag (according to Equation (45)) leads to flow localization, as shown in Figure 25, thus confirming the crucial role that this drag force plays in $2 \mathrm{D}$ foam rheology.

Localization lengths $l_{1 / 10}$ were extracted from such data as the distance away from the moving boundary where the velocity has decayed to $1 / 10$ of that at the boundary, see Equation (20). Figure 26 shows the variation of $l_{1 / 10}$ as a function of Deborah number $D e$ (as defined in Equation (46)); the data is well described by a fit to a power law with $l_{1 / 10} \propto D e^{-0.30}$. This scaling is in excellent agreement with the prediction of the continuum model which gives $l \propto D e^{\frac{a-b}{1+a}}$ (by use of Equation 


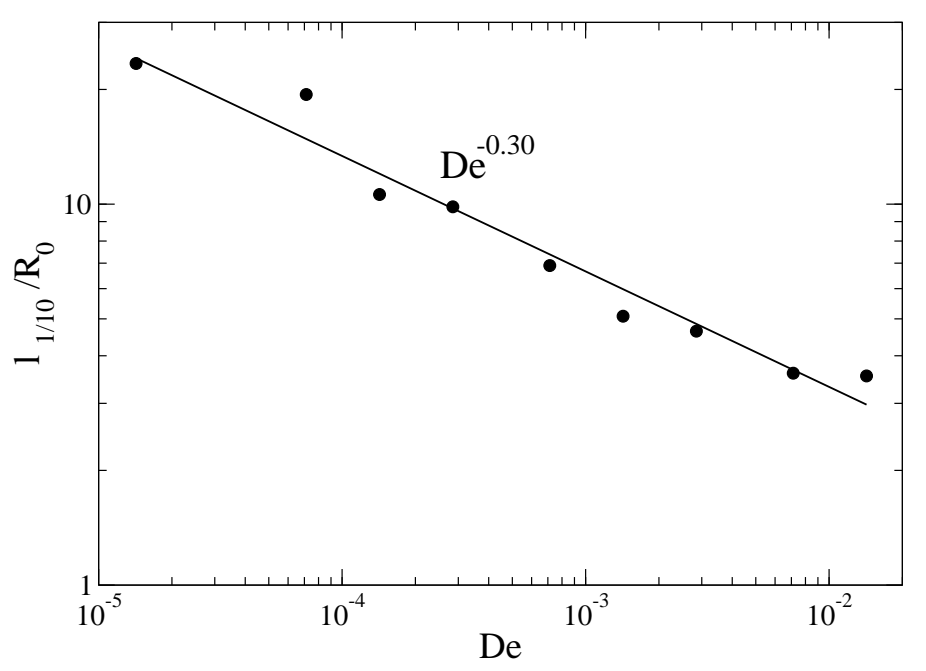

Figure 26. Normalised localisation length $\lambda_{1 / 10} / R_{0}$ as a function of th e Deborah number (and using the definition of Equation (20) for the localization length). The solid line is a fit of the data to a power law, resulting in $\lambda_{\frac{1}{10}} / R_{0}=0.87 D e^{-0.30}$, in excellent agreement with theory. (Here the sample width was $28 R_{0}$ where $R_{0}$ is the mean bubble radius.)

(23) and Equation (10)). Using the determined value of the Herschel-Bulkley index, $a=0.54$, and the wall drag exponent, $b=1$ of the bubble model indeed gives the above scaling relation with exponent -0.30 . Note that there is no free/adjustable parameter in the theory.

In summary, the bubble model simulations have provided remarkably good support for the validity of the continuum model. In addition, the soft disk model was used to explore the question of the significance of the Herschel-Bulkley relation.

\section{The interpretation of the Herschel-Bulkley relation}

We have seen that the soft-disk model has shear localization properties broadly consistent with those of the viscous froth model, the continuum model, and with experiments on real 2D foams. We have noted however, that the value of the HerschelBulkley index $a$ that is required to establish conformity to the predictions of the continuum model is intriguing. Since only linear dissipative forces are incorporated in this version of the disk model, a linear viscous term is rather to be expected, 
i.e. $a=1$, the Bingham model. Instead values of $a \simeq 0.54$ were found in the simulations of [27]. Recall that also the Leiden group found such non-linearity; their experimental data was best described by $a=0.37$ [28], which they could confirm independently. Non-linearity $(a=1 / 2)$ arises also out of the recently introduced "kinetic elastoplastic" (KEP) model for the flow of soft matter with granularity, which incorporates the key features of flow, namely global elastic deformations and local plastic rearrangements [67].

Let us first rehearse the argument that suggests that the observed values of $a$ are anomalous. At least for low shear rates, it is natural to expect some quasistatic regime in which the detailed motion of the constituent bubbles remains the same, merely speeding up as the shear rate is increased. Topological changes are effectively instantaneous, so there is no correlation between their timescale and that of the shearing motion. Then if the viscous forces are linear in velocity, surely the total or average stress resulting from them must also be linear in the strain-rate? The soft disk calculations, having been pursued over three orders of magnitude in strain rate, show that this argument fails.

One seems forced to conclude that the motion of the bubbles is complex [68] and varies in some more singular way, as shear rate decreases to zero. This notion resonates with what has been said about granular materials (e.g. Goldenberg [69]) from time to time.

In the case of the experimental data, complex dynamics is not the only explanation on offer. The group of Denkov [19], in particular, contends that the Herschel-Bulkley index simply corresponds to that of the local viscous forces. Indeed it is well established that neither the wall drag force nor internal dissipative forces scale linearly. Moreover, the power-law indices that they entail depend upon the type of surfactant. Chemistry is not irrelevant!

At the time of writing, this debate is proceeding. What combination of the form of local forces and complex dynamics is responsible for the value of $a$ in each particular case? We leave it here, but will review the forms of the local forces that have been forced upon our attention.

\section{The forms of local forces}

It is sometimes remarked that the forces that operate in a foam are simple. In general this is hardly true. Certainly it cannot be said of the dissipative forces that operate 
in dynamics. And even the elastic force is awkward in the apparently simple model of interacting disks, discussed in section 13, if it is to bear some relation to reality. The simple form used in the soft disk model, a pairwise Hooke's-law force acting upon contact, is certainly not correct near the "jamming" transition of bubbles, or for that matter of elastic particles [70, 71]. But our principal concern here is with the two types of dissipative force which lie behind two of the terms in the continuum theory. Neither takes the obvious elementary form, linear in velocity. We come at last to the significance of $a$ and $b$.

The force associated with the sliding of bubbles along a smooth surface which is wetted by the solution is associated with the name of Bretherton [55] who performed an early theoretical analysis of the problem and succeeded in deriving a power law for the force as a function of velocity,

$$
F \propto v^{n} .
$$

More recent studies, with less approximations and more computation, reach similar conclusions, except that a distinction is made between mobile and immobile interfaces [19], for which it would appear that

$$
\begin{array}{ll}
n=2 / 3 & \text { (mobile) } \\
n=1 / 2 & \text { (immobile). }
\end{array}
$$

Various experiments confirm these indices: recall that $n$ was directly measured in the Leiden experiments for mobile surfaces (section 3.3), resulting in $n=0.67 \pm 0.02$. Some uncertainty remains over the mobile/immobile distinction and the range of validity of these nonlinear forms.

The form of local dissipative forces that act internally is much less clear - indeed their very origin is disputable. It is addressed in the recent work of Denkov et al. $[21]$.

\section{Comparison with granular matter}

Foam may be seen as a prototype for a wide class of complex fluids. The flow of granular materials is of particular interest at present, and bears at least a superficial resemblance to that of foam [72]. Continuum descriptions have not been generally favoured, but have been advocated by some, e.g. [73]. 
2D rheological measurements in granular material have been undertaken by Behringer [74], displaying localization that is similar to that seen in 2D foam.

To analyse the complex of ideas that is adduced in the case of granular media would take us too far afield. See e.g. [75]. Suffice it to say here that the perceived gulf between foamy and granular systems seems to be shrinking and the results that we have reviewed here may soon find applications to grains.

\section{Conclusion}

The general continuum model has proved to be rich in its implications, despite its simplicity. These have included shear localization, its velocity dependence, the non-uniqueness of solutions, history dependence and interesting transient behaviour. The model has proved to be consistent with several experiments and awaits further testing. Its precise relation to quasistatic simulations remains to be defined.

The essential message is very straightforward: wall drag (a general but not inevitable feature of the 2D foam) entails localization. However, its effects may be modified at low boundary velocities, for which the difference of yield and limit stresses becomes significant and indeed can localize shear in the absence of wall drag. We attribute localization in quasistatic simulations to this effect.

In three dimensions there is no obvious counterpart of wall drag. For the explanation of localization in 3D (which is not confined to boundaries) other explanations must be sought. The present work does contain one. Again it is the effect of the yield/limit stress differences (see section 10), but there are surely others.

This study has provoked some of the recent debate on the significance of the power law in the Herschel-Bulkley relation (section 14). In this respect the 2D foam has fulfilled its old promise, to provide a transparent test-bed for ideas and experiments that carry much wider implications.

Finally let us acknowledge another large body of work that has not been included here, though it has been closely associated with some of the experiments and quasistatic simulations that we have mentioned. It relates to the detailed role of topological changes in rheology. Once an interpretation of continuous shear is sought at the cellular level, it is to be found in the local rearrangements that take place. Rather than fracturing or otherwise degrading, the foams cells constantly rearrange. At low rates of shear, individual events ("T1 processes" [76]) consist of simple neighbour-swapping [77]. The pursuit of the statistics of these events and 
their correlations $[54,78,31,35,36,50, ?, ?]$ is an attractive route towards deeper interpretations of some of the topics we have discussed.

\section{Acknowledgments}

Research supported by the European Space Agency (MAP AO-99-108:C14914/02/NL/SH and AO-99-075:C14308/00/NL/SH). We thank V. Langlois and R.J. Clancy for their contributions to this work and A. Kabla for useful discussions. JB acknowledges IRCSET Embark for funding this project. IITAC, the HEA, the National Development Plan and the Trinity Centre for High Performance Computing are acknowledged for the use of the computing facilities at TCD. DW thanks Science Foundation Ireland (08/RFP/MTR1083 STTF 08) for support for a visit to the Cavendish Laboratory, Cambridge, UK, during which the bulk of the review was completed, and the laboratory for its hospitality.

[1] A. M. Kraynik. Foam flows. Ann. Rev. Fluid Mech., 20:325-357, 1988.

[2] C.S. Smith. The shapes of metal grains, with some other metallurgical applications of topology. Metal Interfaces (ASM Cleveland), 1952.

[3] L. Bragg and J.F. Nye. A dynamical model of a crystal structure. Proc. Royal Soc. London Series A, 190:474-481, 1947.

[4] A. Abd El Kader and J.C. Earnshaw. Evolution of a two-dimensional foam containing a single topological defect: An experimental study. Physical Review E, 56, 3251 - 3255(3):3251 $3255,1997$.

[5] M.F. Vaz and M. A. Fortes. Experiments on defect spreading in hexagonal foams. J. Phys. Condens. Matter, 9:8921, 1997.

[6] D. Weaire and S. Hutzler. The physics of foams. Clarendon press, Oxford, 1999.

[7] D.J. Durian. Foam mechanics at the bubble scale. Phys. Rev. Lett., 75:4780-4783, 1995.

[8] D.J. Durian. Bubble-scale model of foam mechanics: Melting, nonlinear behavior, and avalanches. Phys. Rev. E, 55:1739-1751, 1997.

[9] I.K. Ono, C.S. OHern, D.J. Durian, S.A. Langer, A.J. Liu, and S.R. Nagel. Effective temperatures of a driven system near jamming. Physical Review Letters, 89:095703, 2002.

[10] I. Cantat and R. Delannay. Dynamical transition induced by large bubbles in $2 \mathrm{~d}$ foam flows. Phys. Rev. E., 67:031501-031504, 2003.

[11] I. Cantat, N. Kern, and R. Delannay. Dissipation in foam flowing through narrow channels. Europhys. Lett., 65:726-732, 2004.

[12] I. Cantat and R. Delannay. Dissipative flows of 2d foams. Euro. Phys J. E, 18:55-67, 2005.

[13] B. Dollet, F. Elias, C. Quilliet, C. Raufaste, M. Aubouy, and F. Graner. Two-dimensional flow of foam around an obstacle: Force measurements. Phys. Rev. E, 71:031403, 2005.

[14] B. Dollet, M. Aubouy, and F. Graner. Anti-inertial lift in foams: a signature of the elasticity of complex fluids. Phys. Rev. Lett., 95:168303, 2005. 
[15] B. Dollet and F. Graner. Two-dimensional flow of foam around a circular obstacle: local measurements of elasticity, plasticity and flow. J. Fluid Mech., 585:181-211, 2007.

[16] C. Raufaste, B. Dollet, S. Cox, Y. Jiang, and F. Graner. Yield drag in a two-dimensional flow of foam around a circular obstacle: Effect of fluid fraction. Eur. Phys. J. E., 23:217-228, 2007.

[17] F. Graner P. Marmottant, C. Raufaste. Discrete rearranging disordered patterns, part ii: 2d plasticity, elasticity and flow of a foam. Eur. Phys. J. E, 25:371384, 2008.

[18] H. Caps, S. Traberlsi, S. Dorbolo, and N. Vandewalle. Bubble and granular flows: differences and similarities. Physica A, 344:424-430, 2004.

[19] N.D. Denkov, V. Subramanian, D. Gurovich, and A. Lips. Wall slip and viscous dissipation in sheared foams: Effect of surface mobility. Colloids Surf. A, 263:129-145, 2005.

[20] N.D. Denkov, S. Tcholakova, K. Golemanov, K.P. Ananthapadmanabhan, and A. Lips. Viscous friction in foams and concentrated emulsions under steady shear. Physical Review Letters, 100:138301, 2008.

[21] S. Tcholakova, N.D. Denkov, K. Golemanov, K.P. Ananthapadmanabhan, and A. Lips. Theoretical model of viscous friction inside steadily sheared foams and concentrated emulsions. Physical Review E, 78:011405, 2008.

[22] G. Debrégeas, H. Tabuteau, and J.-M. di Meglio. Deformation and flow of a two-dimensional foam under continuous shear. Phys. Rev. Lett., 87:178305, 2001.

[23] E. Janiaud, D. Weaire, and S. Hutzler. Two-dimensional foam rheology with viscous drag. Physical Review Letter, 97:038302, 2006.

[24] R. J. Clancy, E. Janiaud, D. Weaire and S. Hutzler. The response of $2 \mathrm{~d}$ foams to continuous applied shear in a couette rheometer. Eur. Phys. J. E, 21:123-132, 2006.

[25] E. Janiaud, D. Weaire, and S. Hutzler. A simple continuum model for the dynamics of a quasitwo dimensional foam. Colloids and Surfaces A: Physicochem. Eng. Aspects, 309:125-131, 2007.

[26] D. Weaire, S. Hutzler, V.J. Langlois, and R.J. Clancy. Velocity dependence of shear localisation in a 2d foam. Phil. Mag. Lett., 88:387-396, 2008.

[27] V.J. Langlois, S. Hutzler, and D. Weaire. Rheological properties of the soft disk model of 2d foams. Phys. Rev. E, 78:021401, 2008.

[28] G. Katgert, M.E. Möbius, and M. van Hecke. Rate dependence and role of disorder in linearly sheared two-dimensional foams. Phys. Rev. Lett., 101:058301, 2008.

[29] G. Katgert M.E. Möbius and M. van Hecke. Relaxation and flow in linearly sheared twodimensional foams. Physical Review Letters, submitted, 2008.

[30] G. Katgert, A. Latka, M.E. Möbius, and M. van Hecke. Flow in linearly sheared twodimensional foams: From bubble to bulk scale. Physical Review E, 79:066318 (15 pages), 2009.

[31] A. Kabla and G. Debrégeas. Local stress relaxation and shear banding in dry foam under shear. Phys. Rev. Lett., 90:258303, 2003.

[32] A. Kabla and G. Debrégeas. Quasi-static rheology of foams. Part 1. Oscillating strain. Journal of Fluid Mechanics, 587:23-44, 2007.

[33] A. Kabla, J. Scheibert, and G. Debrégeas. Quasistatic rheology of foams. Part 2. Continuous 
shear flow. Journal of Fluid Mechanics, 587:45-72, 2007.

[34] S.J. Cox. A viscous froth model for dry foams in the surface evolver. Coll. Surf. A, 263:81-89, 2005.

[35] A. Wyn, I.T. Davies, and S.J. Cox. Simulations of two-dimensional foam rheology: localization in linear couette flow and the interaction of settling discs. Euro. Phys. J. E, 26:81-89, 2008.

[36] S.J. Cox and A. Wyn. Localization of topological changes in couette and poiseuille flows of two-dimensional foams. AIP Conf. Proc., 1027:836-838, 2008.

[37] P. Schall and M. van Hecke. Shear bands in matter with granularity. Annual Review of Fluid Mechanics, 42:67-88, 2010.

[38] S.J. Cox and E. Janiaud. On the structure of quasi-two-dimensional foams. Philosophical Magazine Letters, 88:693-701, 2008.

[39] Y. Wang, K. Krishan, and M. Dennin. Impact of boundaries on velocity profiles in bubble rafts. Phys. Rev. E, 73:031401, 2006.

[40] M. Dennin. Discontinuous jamming transitions in soft materials: coexistence of flowing and jammed states. J. Phys.: Condens. Matter, 20:283103 (14pp), 2008.

[41] J. Lauridsen, G. Chanan, and M. Dennin. Velocity profiles in slowly sheared bubble rafts. Phys. Rev. Lett., 93:018303, 2004.

[42] C. Gilbreth, S. Sullivan, and M. Dennin. Flow transitions in two-dimensional foams. Phys. Rev. E, 74:051406, 2006.

[43] K. Krishan and M. Dennin. Viscous shear banding in foam. Physical Review E, 78:051504, 2008.

[44] J. Lauridsen, M. Twardos, and M. Dennin. Shear-induced stress relaxation in a twodimensional wet foam. Phys. Rev. Lett., 89:098303, 2002.

[45] J. Goyon, A. Colin, G. Ovarlez, A. Ajdari, and L. Bocquet. Spatial cooperativity in soft glassy flows. Nature, 454:84-87, 2008.

[46] D. Weaire and J.P. Kermode. The evolution of the structure of a two-dimensional soap froth. Phil. Mag. B, 47:L29-L31, 1983.

[47] D. Weaire and J.P. Kermode. Computer simulation of a two-dimensional soap froth. I. Method and motivation. Phil. Mag. B, 48:245-259, 1983.

[48] D. Weaire and J.P. Kermode. Computer simulation of a two-dimensional soap froth. II. Analysis of results. Phil. Mag. B, 50:379-395, 1984.

[49] N. Kern, D. Weaire, A. Martin, S. Hutzler, and S.J. Cox. Two-dimensional viscous froth model for foam dynamics. Phys. Rev. E, 70:041411, 2004.

[50] J.D. Barry, D. Weaire, and S. Hutzler. Shear localisation with 2d visous froth and its relation to the continuum model. Rheologica Acta, submitted, 2009.

[51] T.-L. Fu D. Weaire and J.P. Kermode. On the shear elastic constant of a two-dimensional froth. Philosophical Magazine B, 54:L39-L43, 1986.

[52] F. Bolton and D. Weaire. Rigidity loss transition in a disordered 2d froth. Physical Review Letters, 65:3449, 1990.

[53] F. Bolton and D. Weaire. The effects of Plateau borders in the two-dimensional soap froth. II. General simulation and analysis of rigidity loss transition. Phil. Mag. B, 65:473-487, 1992.

[54] S. Hutzler, D. Weaire, and F. Bolton. The effects of Plateau borders in the two-dimensional 
soap froth, III. Further results. Phil. Mag. B, 71:277, 1995.

[55] F.P. Bretherton. The motion of long bubbles in tubes. J. Fluid Mech., 10:166-188, 1961.

[56] R.J. Hunter. Introduction to Modern Colloid Science. Oxford University Press, UK, 1993.

[57] D. Weaire. The rheology of foam. Current Opinion in Colloid $\& 3$ Interface Science, 13:171-176, 2008.

[58] D. Weaire, R.J. Clancy, and S. Hutzler. A simple analytical theory of localisation in $2 \mathrm{~d}$ foam rheology. Philosophical Magazine Letters, 89:294-299, 2009.

[59] P. Marmottant and F. Graner. An elastic, plastic, viscous model for slow shear of a liquid foam. Eur. Phys. J. E, 23:337-347, 2007.

[60] D. Weaire, R.J. Clancy, and S. Hutzler. The origin of the strain-rate discontinuity in $2 \mathrm{~d}$ foam rheometry with circular geometry. arXiv:090\%.1624v1 [cond-mat.soft], 2009.

[61] J.A. Glazier and D. Weaire. The kinetics of cellular patterns. Journal of Physics: Condensed Matter, 4:1867-1894, 1992.

[62] D. Weaire and S. McMurry. Some fundamentals of grain growth. Solid State Physics, 50:1-36, 1996.

[63] W. Drenckhan, S.J. Cox, G. Delaney, H. Holste, D. Weaire, and N. Kern. Rheology of ordered foams - on the way to discrete microfluidics. Colloids and Surfaces a-Physicochemical and Engineering Aspects, 263(1-3):52-64, 2005.

[64] K. A. Brakke. The surface evolver. Experimental Mathematics, 1:141-165, 1992.

[65] K. Brakke. http://www.susqu.edu/brakke/evolver/evolver.html.

[66] R.G. Larson. The Structure and Rheology of Complex Fluids. Oxford University Press, Oxford, 1999.

[67] L. Bocquet, A. Colin, and A. Ajdari. Kinetic theory of plastic flow in soft glassy materials. Physical Review Letters, 103:036001, 2009.

[68] D. Weaire and S. Hutzler. Foam as a complex system. J. Phys.: Condens. Matter, 21:4227, 2009.

[69] C. Goldenberg, A. P. F. Atman, P. Claudin, G. Combe, and I. Goldhirsch. Scale separation in granular packings: Stress plateaus and fluctuations. Phys. Rev. Lett., 96:168001, 2006.

[70] D.C. Morse and T.A. Witten. Droplet elasticity in weakly compressed emulsions. Europhysics Letters, 22:549, June 1993.

[71] M. D. Lacasse, G. S. Grest, and D. Levine. Deformation of small compressed droplets. Physical Review E, 54(5):5436-5446, 1996.

[72] D. Weaire, V. Langlois, M. Saadatfar, and S. Hutzler. Foam as granular matter. In Di Matteo T Aste T and Tordesillas A, editors, Granular and Complex Materials, World Scientific Lecture Notes in Complex Systems, volume 8, pages 1-26, New Jersey, 2007. World Scientific Publishing.

[73] G. Picard, A. Ajdari, F. Lequeux, and L. Bocquet. Complex spatiotemporal behavior within a simple elastoplastic model. Phys. Rev E, 71:010501, 2005.

[74] R.P. Behringer. Fluctuations in granular materials. In Di Matteo T Aste T and Tordesillas A, editors, Granular and Complex Materials, World Scientific Lecture Notes in Complex Systems, volume 8, pages 187-215, New Jersey, 2007. World Scientific Publishing.

[75] P.-Y. Lagrée and D. Lhuilliera. The couette flow of dense and fluid-saturated granular media. 
European J. Mech. B/Fluids, 25:960-970, 2006.

[76] D. Weaire and N. Rivier. Soap, cells and statistics: Random patterns in two dimensions. Contemp. Phys., 25:59, 1984.

[77] M. Durand and H.A. Stone. Relaxation time of the topological t1 process in a two-dimensional foam. Phys. Rev. Lett., 97:226101, 2006.

[78] M. Dennin and C.M. Knobler. Experimental studies of bubble dynamics in a slowly driven monolayer foam. Physical Review Letters, 78:2485 - 2488, 1997. 